\title{
Big Data: Konsequenzen für das Human Resource Management Schweizer Unternehmen
}

\author{
Georg Reissich, Geraldine Rohr, Bernadette Wanzenried \\ und Jochen Schellinger
}

\section{Zusammenfassung}

Die Digitalisierung von Wirtschaft und Gesellschaft hat zur Folge, dass aufgrund der fast unbegrenzten Kommunikations- und ökonomischen Transaktionsmöglichkeiten mit einer Vielzahl technischer Zugangsmöglichkeiten rund um die Uhr in- und außerhalb von Unternehmen kontinuierlich Daten generiert werden. Die hinter diesen Daten stehenden Informationen können für Unternehmen sehr wertvoll werden, sei es als Grundlage neuer Geschäftsmodelle, bei der Weiterentwicklung ihrer Produkte oder zur Optimierung interner Prozesse. Die riesige Datenmenge und zugehörige neue Analysetechniken können unter dem Oberbegriff „Big Data“ zusammengefasst werden. Der Beitrag untersucht den Einfluss von Big Data auf das Human Resource Management Schweizer Unternehmen. Im Rahmen einer qualitativen Untersuchung wurden thesenbasierte Interviews mit Expertinnen und Experten von Unternehmen und Institutionen geführt, die für die Zukunft einen zunehmenden Einsatz von Big-Data-Analysen auch im Human Resource Management vermuten lassen, auch wenn derzeitige Anwendungen noch eher rudimentär erfolgen.

G. Reissich · G. Rohr · B. Wanzenried

Bern, Schweiz

J. Schellinger $(\square)$

Berner Fachhochschule Wirtschaft, Bern, Schweiz

E-Mail: jochen.schellinger@bfh.ch 


\subsection{Einleitung}

In der Unternehmenswelt ist die Digitalisierung weit vorangeschritten. Mit dem Begriff „Big Data“ zeichnet sich eine neue Stufe der digitalen Entwicklung ab. In vielen Unternehmensbereichen bestehen bereits umfangreiche Erfahrungen im Umgang mit großen Datenmengen, im Personalwesen wurde das Thema bislang aber offenbar eher zurückhaltender angegangen. Dies könnte unter anderem an dem konservativ ausgelegten Arbeitnehmerschutz in der Schweiz liegen (Hofmann und Meyer 2017) oder auch daran, dass die Thematik im Bereich des Human Resource Managements (HRM) nur ein geringeres Nutzenpotenzial aufweist. Die Auswirkungen und Anwendungen von Big Data in den Personalabteilungen Schweizer Unternehmen sind aktuell wenig bis gar nicht erforscht. Vor diesem Hintergrund werden in einem ersten Schritt der Untersuchung zunächst der Status quo zur Thematik in der Literatur aufgezeigt. Anschließend werden die Ergebnisse der eigenen empirischen Untersuchung zur Big-Data-Praxis Schweizer Unternehmen vorgestellt. Die gewonnenen Erkenntnisse dienen als aktuelle Standortbestimmung und helfen dabei, einen Blick in die Zukunft der Big-Data-Anwendung im HRM zu werfen.

Die theoretisch-konzeptionelle Analyse bildet die Grundlage für die Formulierung der Leitthesen für die empirische Untersuchung. Die Bestandsaufnahme der Literatur orientiert sich an folgenden Orientierungsfragen:

- Ist Big Data überhaupt ein relevanter Trend für das HRM in Schweizer Unternehmen?

- Welche Anwendungsoptionen gibt es bereits und welche sind für die Zukunft noch zu erwarten?

- In welchem Ausmaß wird Big Data das HRM in Unternehmen verändern?

- Welche Chancen und Risiken sind mit Big Data im HRM verbunden?

- In welchem Umfang ist Big Data bereits im HRM in der Schweiz angekommen?

- Gibt es im Schweizer Kontext spezifische rechtliche und ethische Aspekte im Thema?

Im zweiten Teil der Untersuchung werden mittels einer qualitativen Befragung eines breit aufgestellten Portfolios an Interviewpartnerinnen und -partnern die als Leitthesen formulierten abgeleiteten Annahmen überprüft.

\subsection{Digitalisierung - Gegenstand und Ausprägungsformen}

Allgemein bezeichnet der Begriff Digitalisierung im ursprünglichen Sinne das Umwandeln von analogen Werten in die digitale Form. Heute hat der Begriff aber noch deutlich weitergehende Bedeutungen. Er steht unter anderem auch für die Überführung von Information und Kommunikation in die digitale Modifikation von Instrumenten, Geräten oder Fahrzeugen oder wird als Synonym für die ,digitale Revolution“ verwendet, die auch als „,vierte industrielle Revolution“ bzw. „digitale Wende“ bezeichnet wird. Während sich die Digitalisierung bis in die 1990er-Jahre primär mit der Automatisierung und Optimierung 
von Privathaushalten, Arbeitsplätzen und Prozessen auseinandergesetzt hat, stehen seit Beginn des 21. Jahrhunderts disruptive Technologien und innovative Geschäftsmodelle sowie Autonomisierung, Flexibilisierung und lndividualisierung in der Digitalisierung im Vordergrund (Bendel 2018b).

Der schnelle Fortschritt der digitalen Transformation wird von Expertinnen und Experten unter anderem mit einem Generationenwechsel in der Arbeitswelt verbunden. Führungskräfte und Mitarbeitende sind zunehmend vertrauter mit den Möglichkeiten, die sich ihnen mit der Digitalisierung bieten. Auch in der Politik ist das Thema Digitalisierung angekommen. So wurde etwa im Jahr 2017 unter der Leitung des damaligen Bundespräsidenten Johann Schneider-Ammann von etwa 50 Unternehmerinnen und Unternehmern, Wissenschaftlerinnen und Wissenschaftlern sowie Politikerinnen und Politikern ein „Digitales Manifest für die Schweiz“ entwickelt. Dieses steht für eine gemeinsame visionäre Stoßrichtung bei der Digitalisierung der Schweiz unter Berücksichtigung möglichst aller Interessengruppen (Schneider-Ammann et al. 2017).

In Bezug auf die Digitalisierung in der Arbeitswelt sind interne und externe rahmengebende Faktoren zu unterscheiden. Externe Rahmensetzungen können nur bedingt oder indirekt von Unternehmen beeinflusst werden. Es handelt sich hierbei um relevante Aspekte der Infrastruktur, der Politik, der Gesetzgebung, der Gesellschaft und sich verändernder Kundenanforderungen. Interne Faktoren liegen weitgehend im Gestaltungsbereich der Unternehmen und umfassen beispielsweise die Mitarbeitendenqualifizierung, die Demografie der Belegschaft, die Unternehmenskultur oder die Finanzsituation (Schneider-Ammann et al. 2017; Meier und Franke 2016). In diversen Praxisbeispielen aus der DACH-Region wird deutlich, dass diese Faktoren, abhängig von der Branche, unterschiedlich stark ausgeprägten Einfluss nehmen (Beck et al. 2016).

Die Digitalisierung konkretisiert sich in verschiedenen Ausprägungsformen. Die wichtigsten technologischen Trends, die mit der Digitalisierung gemeinhin verbunden werden, sind Industrie 4.0, Internet of Things, Cloud Computing, künstliche Intelligenz und Big Data.

Nachdem mit der dritten industriellen Revolution die Automatisierung den Arbeitsalltag grundlegend verändert hat, wird unter dem Begriff Industrie 4.0 ein weiterer digitalisierungsbedingter Paradigmenwechsel für die Wirtschaft gesehen. Mit ihr geht eine Verschmelzung und systemübergreifende Zusammenführung von digitalen, physischen und biologischen Sphären einher, bei der die reale und die virtuelle Welt immer mehr zusammenwachsen (Siemens AG 2013).

Viele auch noch so kleinen Geräte und Maschinen sind heute von ihrer technischen Ausstattung her Computer, welche sich mit dem Internet verbinden und miteinander interagieren können. Dieser virtuelle Kontakt von physischen Gegenständen wird als Internet of Things (IoT) bezeichnet (Siepermann 2018). In der Unternehmenspraxis werden beispielsweise heute Produktionsmaschinen eingesetzt, die technische Störungen oder Materialengpässe automatisch detektieren und melden und im Bereich der Logistik werden in digitalisierten Lagerhallen die Bestände automatisch überwacht und Folgebestellungen bedarfsabhängig ausgelöst. In der Landwirtschaft kommen Messgeräte zum Einsatz, die 
Boden- und Wetterbedingungen für die Landwirtinnen und Landwirte kontinuierlich auswerten und Handlungsbedarfe signalisieren (Boorman 2017). Kritiker dieser Entwicklungen sehen mit dem technischen Fortschritt und der zunehmenden Automatisierung und Autonomisierung von Maschinen und Gegenständen die Gefahr, dass die Menschen die Kontrolle über die Produktions- und Logistiksysteme zunehmend verlieren (Lindner 2018).

Wesentliches Merkmal des Cloud Computing ist, dass Dateien, Programme oder Dokumente nicht mehr auf dem eigenen Server der Nutzerinnen und Nutzer, sondern auf einer virtuellen, mit dem Internet verbundenen Umgebung, der „Cloud“, gespeichert werden. Für die Nutzerinnen und Nutzer ergeben sich daraus diverse Vorteile, in erster Linie sind dies aber reduzierte Kosten. Die Risiken des Cloud Computing liegen insbesondere bei der Sicherheit. Sämtliche Dateien, welche in der Cloud gespeichert werden, sind online und somit exponiert für eventuelle Hackerangriffe. Selbst wenn die Daten nicht illegal missbraucht werden, stellt sich bei dieser Technologie die Frage nach Datenschutzregulierungen und Grauzonen der Datennutzung durch die Cloud-Anbieter (Minnich 2020).

Dass Computer Daten automatisch auswerten ist schon lange Alltag. Durch von Expertinnen und Experten vordefinierte Handlungsvorschriften, sogenannte Algorithmen, wird das Analyseverhalten der Geräte definiert und gesteuert. Bei der künstlichen Intelligenz (KI) werden die Aktionen der Computer nicht mehr von Menschen gesteuert, sondern weitestgehend automatisiert. Das Ziel der KI ist es, dass Computer eigenständig mit Menschen kommunizieren, Probleme lösen und Entscheidungen treffen und somit das Leben der Nutzerinnen und Nutzer radikal vereinfachen. Maschinelles Lernen soll durch die Nachahmung biologischer Prozesse in den neuronalen Netzen des menschlichen Gehirns ermöglicht werden (German Research Center for Artificial lntelligence 2016). „Schwache $\mathrm{KI}^{\prime}$ ist auf klare Anwenderprobleme hin programmiert und funktioniert in der menschlichen Wahrnehmung technisch und mathematisch. Sie erfüllt klar definierte Aufgaben und lernt reaktiv, ohne dabei in die Tiefe zu gehen und selbstständige Schlüsse zu ziehen (Moeser 2017). Computersoftware zur Spracherkennung, Navigation oder Texterkennung fällt unter diese Kategorie der schwachen KI. Ein eindeutiges Merkmal der schwachen KI ist, dass sie ihre Schlüsse nicht auf andere Bereiche übertragen kann. Diese intellektuelle

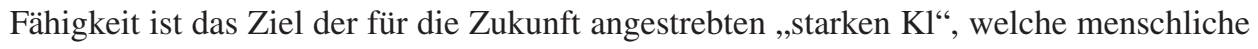
lntelligenz erreichen oder sogar übertreffen soll. Die starke $\mathrm{Kl}$ agiert nicht reaktiv, sondern eignet sich selbstständig Wissen an und entwickelt eigenständig logische Denkstrukturen, Entscheidungs- und Planungsprozesse (Moeser 2017). Kritiker der KI befürchten, dass Maschinen irgendwann so weit entwickelt werden, dass sie die Kontrolle über die Menschen übernehmen könnten (Kappes 2016). Ein weiterer Kritikpunkt, dessen Relevanz sich schon länger und in wachsendem Maße auch real abzeichnet, ist, dass intelligente Maschinen zunehmend die Aufgabe von Arbeitnehmenden übernehmen können und diese ersetzen (Schieb 2017).

Eine weitere zentrale Ausprägungsform und Perspektive der Digitalisierung, die im Fokus dieses Beitrags steht, ist „Big Data“. Diese wird nachfolgend eingehender beschrieben. 


\subsection{Big Data als Digitalisierungsperspektive}

Mit der fortschreitenden Digitalisierung der Gesellschaft erscheint das Erheben von Daten nicht mehr als bloße Notwendigkeit, um eine Produkteigenschaft ausführen oder unterstützen zu können, sondern wird vielfach zu einem parallelen oder gar Hauptgeschäftszweig eines Unternehmens. Seit der Erfindung der Datenspeicherung via Lochkarten im neunzehnten Jahrhundert sind die zu bewältigenden Datenvolumen kontinuierlich und exponentiell angestiegen (Dietzfelbinger et al. 2014). Allein seit 2018 hat sich das weltweite Datenvolumen von ca. 33 Zettabytes bis 2020 auf über 55 Zettabytes erhöht und bis 2025 ist aufgrund einer zunehmenden sensorgesteuerten Automatisierung mit 24/7-Datengenerierungen mit einem weiter zunehmenden Wachstum auf ca. 175 Zettabytes zu rechnen. Die Daten werden dann voraussichtlich zu ca. $50 \%$ in der Cloud gespeichert und etwa ein Drittel der Daten werden real-time Prozess- und Entscheidungsunterstützungsdaten sein (Kroker 2018). Zur effizienten und zielgerichteten Bearbeitung dieser riesigen Datenmengen (Big Data) braucht es heute eine Vielzahl verschiedener Technologien. In der Literatur steht der Begriff Big Data für große Datenmengen, die anhand von speziellen Lösungen gespeichert, verarbeitet und ausgewertet werden (Bendel 2018a). Plattner (2017) betrachtet Big Data als ein Synonym für die Relevanz großer Datenmengen in unterschiedlichen Anwendungsfeldern und die hiermit einhergehende Herausforderung der Verarbeitbarkeit dieser Daten. Es gibt zwar bis heute keine allgemein etablierte Definition von Big Data, aber die beiden Begriffsbeschreibungen können gut als Annäherung für die Grundzielrichtungen anderer definitorischer Ansätze verwendet werden.

Im vorliegenden Beitrag steht Big Data für die Erhebung, Sammlung sowie Speicherung von großen Datenmengen. Ergänzend werden unter „Big Data Analytics“ die analytische Bearbeitung und die Schlussfolgerungen basierend auf diesen Daten verstanden.

Big Data lässt sich anhand von drei idealtypischen Merkmalsausprägungen noch weiter konkretisieren. Das erste Attribut Volume beschreibt die bereits erwähnte hohe Menge an vorhandenen Daten, welche es zu speichern, zu verarbeiten und auszuwerten gilt. Nicht nur Texte, Fotos und Videos, sondern auch Stimmen und sogar Gerüche können in Zahlen übersetzt und digital erfasst werden. Wissenschaftlerinnen und Wissenschaftler sehen sich in diesem Kontext mit diversen Fragen konfrontiert, beispielsweise wie kann bei einer immer größer werdenden Datenmenge der Überblick behalten und eine systematische Verwaltung gewährleistet werden? Auch die Frage danach, ob, wie und wie lange die erforderlichen physischen Geräte mit dem Wachstum des Datenvolumens mithalten können, beschäftigt die Forschung.

Velocity beschreibt die Geschwindigkeit, mit der die Daten generiert und transportiert werden. Ein Beispiel hierfür ist die Foto-Uploadrate von Facebook, die 2015 bereits bei täglich 900 Mio. Fotos lag, die von Facebook-Nutzerinnen und -Nutzer auf die Plattform hochgeladen wurden (Miller 2015). In 2018 hatten die ca. 2,3 Mrd. monatlichen FacebookNutzer bereits etwa 250 Mrd. Fotos hochgeladen (Smith 2020). Wie beim Datenvolumen stellt sich bei der Geschwindigkeit die Frage, wie lange die Technik mit dem hohen Wachstum mithalten kann. Neben der Anwenderfreundlichkeit hat die Geschwindigkeit, 
mit der Daten verarbeitet werden, eine hohe Relevanz auch für die Cybersicherheit. Bei Spam oder Cyberattacken versteckt sich die schädliche Software in einem Fluss an Daten, welcher durch die Firewall eines Gerätes fließt. Diese muss die einzelnen Elemente untersuchen und auf Anomalien prüfen. Je schneller Daten auf sie einschießen, desto schwieriger fällt es ihr, die „Feinde“ zu detektieren (Gewirtz 2018).

Variety beschreibt die Vielfalt der Informationen. Text, als Quelle der digitalen Information, kommt aus einer Vielzahl an oft unstrukturierten Quellen: E-Mails, Zeitungen, Kommentare, Bücher, Software, Social Media, Chatverläufe etc. Das gleichzeitige systematische Erfassen und Auswerten aller Datentypen ist unmöglich, da zu viele Diskrepanzen vorhanden sind: Datenbanken, welche von Menschen bearbeitet werden, sind selten fehlerfrei. Ein weiteres Problem sind technische Unterschiede bei der Datenverarbeitung in Abhängigkeit von den Entwicklern, sodass unterschiedliche Browser, Textverarbeitungsprogramme oder Apps mit einem identischen Satz sehr unterschiedlich umgehen. Es ist somit eine der großen technischen Herausforderungen beim Bearbeiten von Big Data, dass zusammengehörige Informationen von den Algorithmen auch als solche entdeckt und klassifiziert werden (O'Reilly 2012).

Der größte Nutzen von Big Data für Unternehmen liegt im hohen Potenzial zur Unterstützung für die rationale Entscheidungsfindung bei einer Vielzahl von strategischen und operativen Problemstellungen: Anhand von historischen Daten werden Muster erkannt und mögliche Szenarien für die Zukunft aufgezeigt. Diese können anschließend zur Fundierung der Entscheidungsfindung herangezogen werden. In der Praxis hat sich gezeigt, dass sich bereits digitalisierte interne Abläufe durch gezielte Datenauswertungen erheblich verbessern lassen. Dabei können Durchlaufzeiten und/oder Kosten reduziert werden. In datenbasierten Kundenanalysen können Informationen über Kunden gewonnen werden, die weit über die bisher geläufigen demografischen Eckdaten hinausgehen. Detaillierte Kundenprofile erleichtern die Segmentierung und ermöglichen Firmen, ihre Aktionen in Bereichen wie Marketing, Service oder Pricing genauer auf den einzelnen Kunden hin anzupassen (Columbus 2016). Eine analoge Argumentationslogik lässt sich auch auf die Mitarbeitendendaten eines Unternehmens übertragen, die unter Einsatz von Data-Analytics-Algorithmen segmentierte und individualisierte entscheidungsrelevante Informationen für das HRM liefern können.

In Bezug auf den Entwicklungsstand von Big Data gibt die Studie von Seufert (2014) einige Hinweise. Die Studie hebt hervor, dass die befragten Unternehmen den Kostenfaktor bzw. die Kosten-Nutzen-Relation für die Nutzung von Big Data als kritisch bewerten. Erwartungsgemäß ist das themenbezogene Know-how in Großunternehmen deutlich stärker vorhanden als in Klein- und mittelständischen Betrieben, die die schwierige Akquisition von entsprechend qualifiziertem Personal als wichtiges Hemmnis sehen. Das größte Nutzungspotenzial von Big Data wurde vor allem in den Bereichen Management, Unternehmenssteuerung sowie im Vertrieb und Marketing ausgemacht. Das geringste Nutzungspotenzial wurde für den IT-Bereich selbst identifiziert. Big Data hat demnach vor allem in Unternehmensbereichen Einsatzpotenziale, die bereits heute stark mit dem Auswerten von Kennzahlen und Statistiken beschäftigt sind (Seufert 2014). Einer Befra- 
gung der BARC Schweiz GmbH zufolge gehen Unternehmen, bei denen Big Data bereits in die Unternehmensprozesse eingebunden ist, von konkreten Umsatzsteigerungen von $8 \%$ und Kostensenkungen von $10 \%$ aus (Stauffer 2017). Die Studie sieht insbesondere die Bereiche Marketing, Vertrieb und Controlling als Hauptanwendungsgebiete für Big Data, das Personalwesen wurde von den 423 Befragten als am wenigsten bearbeitetes Analysefeld ausgewiesen. Die Untersuchung hebt ferner die hohe Bedeutung von Datenschutz- und Datensicherheitsaspekten als mögliche Anwendungshindernisse hervor (Stauffer 2017). Dieser Aspekt wird durch die immer noch andauernde Revision des Bundesdatenschutzgesetzes (Inkrafttretung voraussichtlich 2021) sowie die Umsetzung der EU-Verordnung zum Datenschutz, die seit Mai 2018 in Kraft ist, als wichtige Rahmensetzungen stark beeinflusst.

\subsection{Big Data und Human Resource Management}

Wie alle anderen Bereiche der Gesellschaft und der Arbeitswelt hat die Digitalisierung auch das Human Resource Management fundamental verändert. Aspekte wie Prozessgestaltung, Arbeitsinstrumente oder Kommunikationstechnologien werden digitalbasiert neu gestaltet und unterstützen das HRM auf dem Weg in eine vernetzte, dynamische und globalisierte Welt. Das Ausmaß der Nutzungsmöglichkeiten digitaler Optimierungen im HRM hängt von Faktoren wie der Größe und dem Geschäftsmodell des Unternehmens ab. So ist anzunehmen, dass eine internationale Großbank mit tausenden Mitarbeitenden über ganz andere Möglichkeiten der Mitarbeitendendatengenerierung und -analyse verfügt, als ein kleineres mittelständisches Unternehmen. Eine Studie zum Entwicklungsstand der Digitalisierung Schweizer Unternehmen, die auch mitarbeitendenbezogene Aspekte untersucht hat, ist die Untersuchung von Greif et al. (2016). In der Studie wurde, entgegengesetzt zur bereits angeführten BARC-Studie, vom Gros der befragten Unternehmensvertreterinnen und -vertreter der Bereich „Mitarbeiter und Kultur“ resp. der dahinterstehende Personalbereich als der am stärksten digitalisierte Unternehmensbereich angeführt.

\subsubsection{Digitalisierung im Human Resource Management}

Nachfolgend werden in der Literatur thematisierte wesentliche Auswirkungen der Digitalisierung auf folgende Hauptaufgabengebiete des Human Resource Managements angeführt: Personalrekrutierung, Personalkommunikation, Personaladministration, Personalstrategie, Personalentwicklung und Changemanagement.

\section{Personalrekrutierung}

Die Anstellungsprozesse sind im modernen HRM bei einem Großteil der Unternehmen weitgehend digitalisiert. In vordigitalen Zeiten lief ein Rekrutierungsprozess im Regelfall wie folgt ab: Die Stelle wurde in Printmedien oder über eine Agentur ausgeschrieben. Die 
Interessentinnen und Interessenten schickten danach ihre Bewerbungsunterlagen zur Prüfung und wurden bei Eignung zu einem oder mehreren Gesprächen, gegebenenfalls auch zu einem Assessment-Center eingeladen. Nach der Zusage wurden die Vertragsunterlagen auf Basis der eingegangenen Papierunterlagen erstellt und die Personaldaten im HRSystem erfasst. Digitalisierte Rekrutierungsprozesse vereinfachen den nun weitgehend papierlosen Prozess für Interessentinnen und Interessenten stark, führen insgesamt aber zu einer höheren Komplexität der Abwicklung. Abb. 11.1 zeigt kontrastierend hierzu exemplarisch einen idealtypischen digitalen Rekrutierungsprozess auf, wie er heutzutage von vielen Unternehmen schon realisiert wird.

Die weitreichende Digitalisierung des Rekrutierungsprozesses führt üblicherweise zu einer deutlichen Effizienzsteigerung bei der Personalbeschaffung und erhöht die Reichweite und Zielgenauigkeit der Personalauswahl. Sie wird außerdem von qualifizierten Bewerbenden in zunehmendem Maße auch erwartet. Im Rahmen der Rekrutierung werden neu eine Fülle von Daten generiert, die für die Auswahlentscheidungen herangezogen werden können und im Falle von Zusagen auch direkt in den Personaldatenstamm des Unternehmens Eingang finden (vgl. dazu auch Hunkeler 2019). Nebst den technischen und administrativen Tätigkeiten gibt es nach wie vor aber auch im digitalen Prozess Aufgaben, welche durch Menschen ausgeführt werden. Die Selektion der Kandidierenden wird zwar von den zur Verfügung stehenden Informationen unterstützt, aber immer noch stark durch menschliche Faktoren wie Intuition und Sympathie geprägt, die vor allem im persönlichen Vorstellungsgespräch wirksam werden.

Bewerbung wird ausgeschrieben (Firmenwebsite, Intern, Online-Jobbörsen)

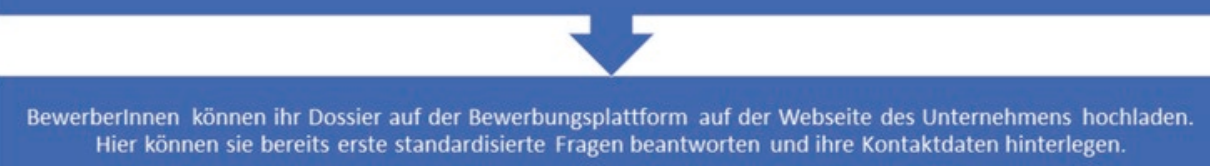
Hier können sie bereits erste standardisierte Fragen beantworten und ihre Kontaktdaten hinterlegen.

Das Unternehmen hat dank der Bewerbungsplattform eine systematische Auflistung aller Kandidierenden. Kandidierende, welche den Anforderungen nicht entsprechen, werden automatisierte Absagen per E-Mail zugestellt.

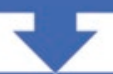

Kandidierenden, welche die Vorselektion überstanden haben, erhalten von dem Unternehmen die Login-Daten für einen Onlinetest. Dieser Test soll das Wissen, Fähigkeiten und Kompetenzen testen und eine weitere Selektion ermöglichen. Die Testergebnisse werden automatisch ausgewertet und den Personalmanagerinnen zugestellt.

Die PersonalmanagerInnen prüfen die Bewerbungsdossiers, die Testergebnisse und holen durch Internetrecherche weitere Informationen über die BewerberInnen ein. Vielversprechende Kandidierende werden zu einem Bewerbungsgespräch und ggf. zu einem Assessment Center eingeladen .

Abb. 11.1 Rekrutierungsprozess in der digitalisierten Welt. (Quelle: eigene Darstellung) 


\section{Personalkommunikation}

Die Digitalisierung verändert die Art und Weise, wie Menschen miteinander kommunizieren, fundamental, privat wie auch in der Arbeitswelt. Den Durchbruch in der digitalen Kommunikation brachte die Einführung der E-Mail, eine Vielzahl weiterer digitaler Kommunikationskanäle folgte. Die neuen Kommunikationsinstrumente ändern nicht nur die Methodik, sondern auch die Sprache, Formalität und Frequenz der Kommunikation. Früher lange, formelle Schriftwechsel werden durch kürzere, informellere Nachrichten reduziert, welche dank Messengersystemen in Kurz- oder Echtzeit zu gesprächsähnlichen Dialogen werden. Telefonieren über das Festnetz wird zunehmend durch die internetunterstützte Distanzkommunikation ersetzt, die Gruppen- und Videoanrufe ermöglicht. Diese sind vermehrt auch Bestandteil ganzheitlicher Kollaborationsplattformen wie zum Beispiel MS Teams. Die Kommunikation im und zwischen Unternehmen verändert sich von einem früher vorherrschenden Top-down- und One-to-all-Informationsfluss hin zu einer Many-to-many-Situation, in der sich Mitarbeitende jenseits von Arbeitsort, Hierarchie oder Funktion jederzeit austauschen können (Dörfel 2013). Die Unternehmen profitieren von dieser Entwicklung und der hiermit verbundenen erleichterten Wissensbeschaffung langfristig durch reduzierte Kosten, effizientere Prozesse und Qualitätssteigerungen bei Produkten oder Services (Institut für angewandte Arbeitswissenschaft 2016). Aus den neuen Formen der digitalen Kommunikation resultieren auch Herausforderungen für die Kommunikation des Personalbereichs nach innen und außen, die berücksichtigen muss, dass mitunter sensible personalbezogene Informationen sich in kürzester Zeit auch ungewollt verbreiten können. In Bezug auf die Sicherheit personalbezogener Daten gilt vor diesem Hintergrund auch, dass hohe Anforderungen an die Administration der Zugriffsberechtigungen zu stellen sind, um die Gefahr eines Missbrauchs zu minimieren (Dörfel 2013).

\section{Personaladministration}

Wie in anderen Unternehmensbereichen auch wurden die administrativen Tätigkeiten und die dahinterstehenden Prozesse des HRM in den vergangenen Jahren weitestgehend digitalisiert. Dies bedeutet beispielsweise, dass Archive und Datenbanken elektronisch bewirtschaftet werden oder dass Gehaltsberechnungen und -auszahlungen in hohem Maße automatisiert erfolgen. Viele Unternehmen streben auch im Personalbereich einen weitgehenden Paperless-Ansatz an, der die Abwicklung möglichst aller betrieblichen Prozesse in elektronischer Form zum Ziel hat (Kefron Limited 2012). Zwischenzeitlich gibt es neben den etablierten großen Anbietern von HRM-Software (z. B. SAP, Oracle, Sage) auch eine Vielzahl kleiner und mittlerer Anbieter von Systemlösungen für alle Unternehmensgrößen und Branchen.

\section{Personalstrategie}

Der heute nahezu unbegrenzte Zugang zu digitalen Informationen ermöglicht auch bei der Entwicklung der Personalstrategie eine wesentlich aktuellere und fundiertere strategische Analyse und Planung auf der Grundlage interner und externer Daten. Für die Durchführung der strategischen Planung kann auf eine Vielzahl von IT-basierten Planungstools 
zurückgegriffen werden. Ein Beispiel hierfür sind etwa umfassende HR-bezogene Datawarehouse-Analyseoptionen in etablierten ERP-Systemen wie SAP. Die Digitalisierung an sich wird aber zunehmend auch selbst inhaltlich zum Gegenstand der strategischen Personalplanung. Die mit der Digitalisierung einhergehenden flexibleren, agilen und vermehrt auch interdisziplinären Arbeitsformen und veränderte Berufsbilder und Rollen führen zu gewandelten Anforderungsprofilen bei den Mitarbeitenden. Dies muss in die Personalstrategie Eingang finden. Ein Beispiel für ein fokussiertes und explizites Aufgreifen der Digitalisierung und der mit ihr einhergehenden Transformationsprozesse in der Personalstrategie ist die jüngst von der Bundesverwaltung verabschiedete „Personalstrategie Bundesverwaltung 2020-2023“ (Eidgenössisches Personalamt EPA 2019).

\section{Personalentwicklung}

Es ist ein Ziel jedes Unternehmens, qualifizierte, motivierte und effizient arbeitende Mitarbeitende zu haben, die sich mit hohem Commitment für das Unternehmen engagieren. Die dafür notwendige kontinuierliche Förderung der Mitarbeitenden ist ein zentraler Punkt eines modernen HRM auch in einer digitalisierten Welt. Die Digitalisierung und die hieraus resultierenden Entwicklungsbedarfe für die Kompetenzen der Mitarbeitenden und Führungskräfte sind einerseits Gegenstand aktueller Personalentwicklungsansätze, andererseits ermöglicht die Digitalisierung eine Vielzahl neuer Formen und Zugänge zur Qualifizierung, die vielfach auch sehr kosteneffizient sind. Orts- und zeitunabhängiges und somit sehr individualisierbares E-Learning etwa kann online via Laptop, Tablet oder Smartphone erfolgen und im Sinne eines Blended-Learning durch Webinare oder Face-toFace- Schulungen ergänzt werden (Köpke 2020).

\section{Changemanagement}

Dem HRM kommt in seiner modernen Ausprägung auch eine wichtige Rolle als Partner der Unternehmensleitung bei organisationalen Veränderungsprozessen zu. Digitalisierungsbedingte Veränderungen im Unternehmen bilden hier keine Ausnahme. Die mit der digitalen Transformation einhergehenden neuen Arbeitsprozesse und -strukturen wecken bei vielen betroffenen Mitarbeitenden Ängste, denen entgegengewirkt werden muss. Hier kommt dem HRM im Schulterschluss mit den Leitungspersonen der Linie eine wichtige Bedeutung zu. Gleiches gilt für die Entwicklungsprogramme zum Aufbau der zukünftig erforderlichen Kompetenzen und die Neujustierung begleitender Personalprozesse.

\subsubsection{Databased Human Resource Management}

Angesichts der vielen systemseitigen Datenbestände und Schnittstellen rund um die betrieblichen Personalprozesse stellt sich die Frage, wie die wachsende Fülle an generierten Personaldaten im Sinne von Big Data in Unternehmen genutzt und verwertet werden kann. Es spricht einiges dafür, dass durch entsprechende datenbasierte Entscheidungsprozesse bessere Lösungen resultieren, die zur Generierung von Wettbewerbsvorteilen führen kön- 
nen (Barman 2015). In der Literatur finden sich allerdings nur wenige Hinweise, die für eine fortgeschrittene Anwendung von Big-Data-Ansätzen in Unternehmen sprechen. Vor allem Anbieter von Systemlösungen nutzen den Begriff plakativ zur Vermarktung ihrer Personalsysteme mit integrierten Analysemöglichkeiten. Die riesige Menge an personenbezogenen Daten wie Lebensläufe, Leistungsaufzeichnungen, demografische Angaben oder persönliche Netzwerke in strukturierter, semi- oder unstrukturierter Form spricht jedoch derzeit vor allem für Großunternehmen für ein großes Potenzial zur systematischen Analyse mit den neu verfügbaren technischen Möglichkeiten und Algorithmen, um Maßnahmen zur Effizienz- und Effektivitätssteigerung sowie zur Verbesserung der Arbeitsbedingungen abzuleiten. Mögliche Einsatzoptionen von Big Data im Kontext des HRM sind People Analytics, Workforce Planning und Talent Analytics, deren Anwendungsgrenzen und -bezüge fließend ineinander übergehen, und die nachfolgend exemplarisch beschrieben werden.

Das Verbinden und eingehende Analysieren von Daten aus dem Umfeld des Unternehmens jenseits von einfachen Datenbankabfragen wird als „Analytics“ bezeichnet. In Verbindung mit personenbezogenen Daten spricht man hier auch von People Analytics oder HR Analytics. Der Begriff steht für eine große Zahl an Methoden und Verfahren, die es erlaubt, die Personaldaten aus verschiedenen Perspektiven zu durchleuchten (Berendes et al. 2016). So können beispielsweise im Rahmen der strategischen Personalplanung zeitgleich zurückreichende Reports, prospektive Prognosen und vergleichende Benchmarkinganalysen mit externem Bezug erstellt und miteinander verknüpft werden. Dies erlaubt in einem Planungsmodell die Simulation strategischer HR-Szenarien zur Ableitung einer auf die geplante Unternehmensentwicklung abgestimmten Personalstrategie. Ein Beispiel, wie eine solche auf People Analytics zurückgehende strategische Szenarioplanung unter Heranziehung eines durchgängigen Datenflusses aus den Systemen SAP und Dynaplan erstellt werden kann, findet sich bei Berendes et al. (2016). Das Beispiel zeigt gleichzeitig die enge Verbindung von People Analytics und Workforce Planning auf. Anhand von Big-Data-Auswertungen können HR-Managerinnen und -manager planen, wie die Belegschaft kurz-, mittel- und langfristig und in Bezug auf Anzahl, Qualität und Verfügbarkeit zusammengestellt sein muss, um die Unternehmensziele zu erreichen, sodass die Rekrutierungs- und Personalentwicklungsaktivitäten entsprechend ausgerichtet werden können (Ebert 2017).

Unternehmen werden beim Besetzen von Vakanzen oft mit enorm vielen Bewerbungen überhäuft. Für die HR-Verantwortlichen ist es zeitlich ein extrem hoher Aufwand, die Dossiers alle sorgfältig zu prüfen und vorzusortieren. Gleichzeitig stehen in den von den Bewerbenden zur Verfügung gestellten Unterlagen nur jene Angaben, welche diese freiwillig von sich preisgeben, in der Regel also Lebensläufe, Leistungsaufzeichnungen, demografische Angaben oder persönliche Referenzen. Die Unternehmen sind aber auch an weitergehenden anderen Informationen interessiert, die normalerweise nur mit hohem $\mathrm{Zu}$ satzaufwand beschafft werden können. Mithilfe von Big-Data-Applikationen kann heute das Internet nach sämtlichen Daten zu der jeweiligen Person durchsucht werden, die das Bild auch über ein LinkedIn-Profil hinaus abrunden können. Man spricht in diesem 
Zusammenhang auch von Talent Analytics. Als Quellen hierfür dienen Social-MediaPlattformen, persönliche und andere mit Bewerbenden verbundene Webseiten, Kommentare oder öffentlich zugängliche Chatrooms (Almog 2018). So könnten Rekrutiererinnen und Rekrutierer auf der Basis frei verfügbarer Daten zur Person beispielsweise erfahren, welchen Platzierung eine Kandidatin bei einer Sportveranstaltung erreicht hat, wie sie sich öffentlich zu gesellschaftlichen und politischen Themen in Kommentarspalten äußert oder wie ihre Familienverhältnisse sind. Das sich ergebende abgerundete Profil von Bewerbenden kann die Prognose erleichtern, wie gut Bewerbende zum Unternehmen passen würden und mit welcher Wahrscheinlichkeit eine hohe Verweildauer im Unternehmen zu erwarten ist.

Bei großen Unternehmen mit einer Vielzahl von Hierarchiestufen ist es für das Management nicht mehr möglich, einzelne Mitarbeitende zu kennen, die nicht im Arbeitsalltag regelmäßig mit den Führungspersonen zu tun haben. Durch Workforce Analytics, dem internen Pendant zu Talent Analytics, lässt sich ein genaueres Bild davon erstellen, welche Mitarbeitenden im Unternehmen welche Leistung erbringen, wie sie arbeiten und wie sich ihr Netzwerk ausgestaltet (Almog 2018). Aus intern generierbaren Daten können auch (indirekte) Rückschlüsse auf die Zufriedenheit von einzelnen Mitarbeitenden gezogen werden. Dies ermöglicht eine bisher normalerweise nicht verfügbare Entscheidungsunterstützung auf individueller Ebene, in aggregierter Form aber auch auf Ebene von Abteilungen oder Geschäftseinheiten. Dies würde beispielsweise erlauben, einen hochaggregierten Zufriedenheitsindikator zu definieren und diesen im Sinne eines Frühwarnindikators im Personalbereich zu verwenden.

Die digitalen Veränderungen der letzten zwei Jahrzehnte haben Prozess, Rollen und die Kommunikation im Human Resource Management massiv verändert. Die hiermit verbundenen Effektivitäts- und Effizienzsteigerungen gingen und gehen aber auch mit einer Vielzahl von Herausforderungen einher. Die Intensivierung von Kommunikation und Vernetzung, neue Strukturen und Arbeitsmodelle und bislang unbekannte Datennutzungsmöglichkeiten erhöhen die Komplexität und schaffen neue Planungs- und Entscheidungsbedarfe. Während im Bereich der administrativen und prozessunterstützenden Digitalisierung des HRM von einem zumeist hohen Entwicklungsstand ausgegangen werden kann, werden Analytics-Anwendungsoptionenen von Big Data für das HRM aber in der Literatur bislang noch kaum diskutiert und empirisch allenthalben sporadisch aufgezeigt. Hier scheint eine dynamischere Entwicklung des Themas erst in Gang zu kommen.

\subsubsection{Rahmenbedingungen für die Nutzung von Big Data in der Schweiz}

Nicht alles, was im Personalbereich datenbasiert technisch möglich ist, darf rechtlich auch umgesetzt werden, denn in jedem Fall ist der Schutz der Privatsphäre des Einzelnen zu gewährleisten. Für den Einsatz von Big Data gibt es eine Reihe von einschränkenden rechtlichen nationalen, aber auch internationalen Rahmensetzungen. Darüber hinaus stel- 
len sich bei der Nutzung von Big Data im Mitarbeitendenbereich auch ethische Fragen und es ist der gesellschaftliche und politische Diskurs zum Umgang mit sensiblen Personendaten zu berücksichtigen, einem Thema, das auch seitens der Gewerkschaften einen hohen Aufmerksamkeitswert hat.

Die Zunahme der Berichterstattung über Datenmissbrauch in und von Unternehmen trägt zu einer wachsenden Sensibilisierung der Bevölkerung hinsichtlich des Umgangs mit ihren Daten bei (Gehrmann 2014). Die Schweiz evaluiert bereits seit 2011 eine Revision des Bundesgesetzes über den Datenschutz (DSG), um die Urheberinnen und Urheber der Daten bestmöglich zu schützen (Bundesamt für Justiz BJ 2017). Die Totalrevision sollte ursprünglich 2020 abgeschlossen sein, aktuell ist aber wohl eher von einer Verabschiedung für das Jahr 2021 zu rechnen. Mit dem Inkrafttreten der Datenschutz-Grundverordnung (EU) 2016/679 des Europäischen Parlaments 2018 ist die Schweizer Regierung zusätzlich in Zugzwang geraten. Durch das Schengen-Abkommen ist die Schweiz dazu verpflichtet, diese Verordnung in einem angemessenen Zeitraum zu ratifizieren, um nicht aus dem Abkommen ausgeschlossen zu werden (Bundesamt für Justiz BJ 2017).

Für Big Data und HRM sind primär die aktuellen und zukünftigen Regelungen zu den personenbezogenen Daten von Bedeutung, also Daten, die direkt mit einer Person in Verbindung gebracht werden können (Verordnung (EU) 2016/679 des Europäischen Parlaments und des Rates zum Schutz natürlicher Personen 2016). In diese Kategorie fallen alle Daten, die in direktem Bezug mit dem Namen einer Person genannt werden sowie alle Informationen, die Rückschlüsse auf eine bestimmte Person erlauben (z. B. Adressen, Telefonnummern, Kreditkarteninformationen).

Die europäische Datenschutz-Grundverordnung (DSGVO) konzentriert sich mehrheitlich auf personenbezogene Daten und hebt damit auf das Individuum ab. Auch wenn die Schweiz nicht als Mitgliedstaat der Europäischen Union gilt, ist sie bei der DSGVO an die Europäische Gesetzgebung gebunden (Widmer 2016). Gemäß Artikel drei der DSGVO findet diese sowohl Anwendung bei direkten EU-Mitgliedstaaten als auch bei Unternehmen, welche den Sitz in einem Nicht-Mitgliedstaat haben, jedoch Waren oder Dienstleistungen in einem EU-Staat anbieten (Verordnung (EU) 2016/679 des Europäischen Parlaments und des Rates zum Schutz natürlicher Personen 2016). Sobald ein Unternehmen also seine Produkte und Dienstleistungen auch in der EU anbietet und verkauft, kommen die europäischen Regelungen zum Tragen (Widmer 2016). Personenbezogene Daten fallen nicht nur bei einem Unternehmen-Kunden-Verhältnis an, sondern auch bei einem Arbeitnehmer-Arbeitgeber-Verhältnis. Für eine Gültigkeit des EU-Rechtsrahmens reicht es bereits aus, wenn ein Unternehmen Bewerberinnen und Bewerber aus EU-Mitgliedstaaten anspricht, z. B. mittels Onlinestelleninseraten. In diesen Fällen gelten die gleichen Vorgaben bezüglich Dokumentations- und Auskunftspflicht, wie beim Umgang mit Kundendaten (Stutz 2018).

Die Regierung der Schweiz hat erkannt, dass die Digitalisierung heute zunehmend das Leben seiner Bürger bestimmt. Förderlich hierfür ist sicher die sehr gute Ausgangslage hinsichtlich der Telekommunikationsinfrastruktur sowie der fortgeschrittene Einsatz von Informations- und Kommunikationstechnologien. In der Strategie „Digitale Schweiz“ 
(BAKOM 2018) werden die Leitlinien für das staatliche Handeln vorgegeben und aufgezeigt, wo und wie Behörden, Wirtschaft, Wissenschaft, Zivilgesellschaft und Politik zusammenarbeiten müssen, damit der digitale Transformationsprozess gemeinsam gestaltet werden kann. Mithilfe definierter Grundsätze, Kernziele, Aktionsfelder und einem zugehörigen Dialog soll in Verbindung mit einer schweizweit vernetzten Umsetzungsinstanz dieses Ziel erfolgreich umgesetzt werden. Ein wichtiges Ziel für die Zukunft ist die Entwicklung einer kohärenten und zukunftsorientierten Datenpolitik und einer entsprechenden nationalen Dateninfrastruktur. Ferner soll der Zugang zu digitalen Inhalten verbessert werden und die Bürgerinnen und Bürger sollen Kontrolle über ihre eigenen Daten ausüben können. Um Schutz vor Cyber-Risiken sicherzustellen, sollen außerdem Strukturen und Prozesse langfristig etabliert werden (BAKOM 2018). Der Schutz der Bürgerinnen und Bürger vor Datenmissbrauch ist also auch in Zukunft ein zentrales Ziel der Digitalen Schweiz und somit von hoher Relevanz für den Umgang mit dem Thema Big Data.

Auch wenn Schweizer Gewerkschaften im internationalen Vergleich ein eher geringeres Gewicht im Wirtschaftsgeschehen haben (lediglich $16 \%$ der Schweizer Arbeitnehmerinnen und Arbeitnehmer sind gewerkschaftlich organisiert) (Liniger 2015), so haben sie als zentrale Interessenvertreter der Arbeitnehmerinnen und Arbeitnehmer viel Einfluss auf die Arbeitswelt der Schweiz und ein hohes Interesse an einem sorgfältigen und personalorientierten Umgang mit den Daten der Mitarbeitenden in den Unternehmen. Die Gewerkschaften haben nicht den Anspruch, den digitalen Wandel in den Unternehmen zu stoppen, da dieser zum Erhalt der Arbeitsplätze in der Zukunft erforderlich ist. Sie setzen sich vielmehr dafür ein, ihre Mitglieder auf den Wandel vorzubereiten und diesen mitzugestalten (Schröder 2015; Lampart und Cirigliano 2017). Risiken sieht der Schweizerische Gewerkschaftsbund (SGB), die Dachorganisation der Schweizer Gewerkschaften, vor allem in Branchen wie dem Detailhandel, die einem digitalisierungsgetriebenen strukturellen Wandel unterliegen. Ferner werden Problempotenziale für administrative Aufgaben und industrielle Tätigkeiten sowie für ältere Arbeitnehmende gesehen (Eichhorst et al. 2015). Die Gewerkschaften fordern Maßnahmen zur digitalen Weiterqualifizierung der Schweizer Arbeitnehmerinnen und Arbeitnehmer und zum Schutz der Gesundheit bei zunehmender Verschmelzung von Arbeit und Privatleben im Mobile und Home Office (Pardini et al. 2017). Aus dieser Verschmelzung von Berufs- und Privatsphäre und der Digitalisierung der Kommunikation resultiert eine vereinfachte Leistungskontrolle von Mitarbeitenden, sodass die Gewerkschaften die Notwendigkeit einer Anpassung des Datenschutzes sehen, um auch in Zukunft das Recht auf Schutz der Privatsphäre zu gewährleisten (Pardini et al. 2017). Gemäß einer Studie der Vereinigten Dienstleistungsgewerkschaft (ver.di) in Deutschland (Müller und Kulemann 2017) fühlen sich mehr als $50 \%$ der Arbeitnehmenden durch die Digitalisierung stärker überwacht als in der vordigitalen Arbeitswelt und $60 \%$ der Befragten verweisen auf eine erhöhte Arbeitsbelastung durch die Digitalisierung.

Im intern und extern nutzbaren Big-Data-Pool der Unternehmen besteht ein großer Teil aus Daten, welche sich direkt oder indirekt auf die Menschen und deren Interaktionen beziehen. Beispiele hierfür sind Daten aus sozialen Netzwerken, das wachsende Feld von Gesundheitsverfolgungsdaten, E-Mails sowie weitere Formen von digital erfassten und 
versendeten Textnachrichten, der Verlauf von Suchmaschinennutzungen und vieles mehr. Die Nutzung dieser Daten kann aus ethischer Sicht sehr problematisch sein (Zwitter 2014). Gemäß Zwitter (2014) stehen im Zentrum von Big Data vier ethische Herausforderungen:

1. Es gibt mehr Daten als jemals zuvor.

2. Big Data ist organisch und bildet die Realität digital besser ab als herkömmliche statistische Daten.

3. Big Data ist aufgrund großer Datensets global.

4. Korrelation versus Kausalität (Analysen werten Zusammenhänge über Kausalzusammenhänge).

Schon allein das Fehlen von Wissen darüber, welche Daten tatsächlich wofür erhoben werden, stellt die Datenerzeugenden (Onlinekonsumentinnen und -konsumenten, Handybesitzerinnen und -besitzer, Mitarbeitende) in eine ethisch benachteiligte Position bezüglich Wissen und dem freien Willen. Globale Daten führen zu einem Machtungleichgewicht zwischen Stakeholdern, die zumeist über das notwendige Wissen verfügen, um Intelligenz und Wissen aus Informationen zu generieren. Ebenfalls weisen Big-Data-Korrelationen auf Ursachen hin, bei denen es keine geben könnte. Folglich werden wir anfälliger dafür, dass wir glauben müssen, was wir sehen, ohne den Ursprung zu kennen (Zwitter 2014).

Das Projekt „Abida“ des Bundesministeriums für Bildung und Forschung in Deutschland identifiziert eine materielle, funktionale, kognitive und soziale Dimension der ethischen Problemlage (Selke et al. 2018). In der materiellen Dimension fokussiert man sich auf Aspekte wie den Handel personenbezogener Daten vor dem Hintergrund eines mehr oder weniger sorglosen Umganges aus Sicht der datenerzeugenden Personen, auf die Autonomie der Algorithmen und die Diskussion komfortabler und manipulativer Technologien. Bei der funktionalen Dimension geht es um limitierte Wechselmöglichkeiten und latente Zwänge, unter anderem durch die Vermischung von Korrelation und Kausalbeziehung, unterschiedliche Definitionen von Big Data im Vordergrund und Hintergrund der Gesellschaft, die Intransparenz von Big-Data-Prozessen, datenbasierte Normalitätsdefinitionen und monopolistische Nutzungsweisen. Die kognitive Dimension setzt sich mit individuellen Entgrenzungen auseinander. Es geht hierbei um Entscheidungsverluste bzw. den Verlust der freien Willensentscheidung und damit auch um Kontrollverluste der betroffenen Individuen. Durch Indiskretionen, dem invasiven Eindringen in die Privatsphäre sowie der Möglichkeit von Manipulation (Nudging) entstehen Befürchtungen um den Verlust der Privatsphäre. Die soziale Dimension thematisiert kollektive Versagenszonen: Zahlreiche Informations-, Macht- und Meinungsasymmetrien führen zu Alternativlosigkeit und Abhängigkeitsverhältnissen. Sozial inklusive Formen von Solidarität führen zu Verlust von Solidarität und Leistung wird nur noch anerkannt, wenn sie messbar ist.

Beide dargelegten ethischen Grundperspektiven auf das Thema Big Data machen deutlich, dass ein großer Bedarf für die Festlegung ethischer Standards für Big Data besteht. Folgende maßgebenden Werte sollen gemäß Richards und King (2014) den Anfang für eine Big-Data-Ethik ebnen: 
1. Neue Definition der Privatsphäre: Privatsphäre ist nicht eine Ansammlung von privaten Informationen, die gehütet werden muss, sondern muss als Regel der Information angesehen werden.

2. Wir müssen lernen, dass geteilte private Information trotzdem vertraulich bleiben kann.

3. Big Data verlangt Transparenz.

4. Big Data kann Identität gefährden.

Das Projekt Abida appelliert dazu, mehr Ethik zu wagen und Big Data als einen chancenreichen und nicht abgeschlossenen gesellschaftlichen Lernprozess zu betrachten. Dabei ist die Verantwortung verteilt zwischen Politik, Bildungseinrichtungen, Forschung, Medien und weiteren Akteuren wie die Wirtschaft (Selke et al. 2018).

Bei der Anwendung von Big Data im HRM sind die angeführten gesetzlichen Rahmenbedingungen der Digitalisierung, die auf den Schutz der Privatsphäre von Individuen abheben, zwingend zu berücksichtigen. Politik und Gewerkschaften in der Schweiz stehen der Digitalisierung grundsätzlich offen gegenüber und haben erkannt, dass man deren Voranschreiten nicht verhindern kann, dass es aber sehr wohl eines Rahmens bedarf, der dabei hilft, Missbrauch vor allem auf der Datenebene vorzubeugen und Sicherheit und Rechtsklarheit für die beteiligten Anspruchsgruppen in Wirtschaft und Gesellschaft zu schaffen. Zu diesen Rahmensetzungen gehört über die Rechtsetzung hinaus auch die Entwicklung und Etablierung ethischer Standards für die Generierung und Analyse von Big Data in allen Lebens- und Wirtschaftsbereichen. Die Etablierung von national und international anerkannten ethischen Grundsätzen zum Umgang mit Big Data befindet sich derzeit wohl noch in einer ersten Entwicklungsphase.

\subsection{Empirische Untersuchung}

\subsubsection{Leitthesen für die empirische Analyse}

Auf Grundlage der erfolgten Bestandsaufnahme der Literatur zum Einsatz von Big Data im HRM können nachfolgende Leitthesen für eine qualitative empirische Untersuchung formuliert werden:

1. Unternehmen verfügen nicht über die nötigen Fachkräfte, um Big Data im HRM adäquat anwenden zu können.

2. Es werden primär Stammdaten für HR-Analytics genutzt. Big Data gemäß der gängigen weitaus breiter gefassten Definition kommt nicht oder nur in geringem Umfang zum Einsatz, da die derzeit genutzten Daten mengenmäßig überschaubar sowie statisch und semi-strukturiert sind.

3. Schweizer Unternehmen scheuen den Einsatz von Big Data aus Reputationsgründen.

4. Big Data im HRM wird in Zukunft die Rekrutierung vollautomatisieren und Rekrutiererinnen und Rektrutierer sowie Pesonalvermittlerinnen und -vermittler größtenteils ersetzen. 
5. Unternehmen wissen in Zukunft über potenzielle individuelle Veränderungen (z. B. potenzielle Kündigung oder Krankheit) ihrer Mitarbeitenden deutlich besser Bescheid.

6. Dank der Anwendung von HR-Analytics können Mitarbeitende in Zukunft anhand ihrer Leistungsprofile und Stärken besser eingesetzt und gefördert werden.

7. Dank der Erkenntnisse von HR-Analytics gewinnen die Unternehmen Wissen, das es Ihnen ermöglicht, die Mitarbeitenden zu motivieren.

8. Im „War for Talents“ haben Unternehmen, die Big Data in der Rekrutierung einsetzen, einen Wettbewerbsvorteil.

9. Durch die bislang restriktive Gesetzgebung wird der Fortschritt der Anwendung von Big Data im HRM gehemmt.

10. Die negative Beeinflussung der öffentlichen Wahrnehmung von Big Data durch die Medien führt zu einer verringerten Akzeptanz von Big Data in der Gesellschaft.

Die Leitthesen bilden die Grundlage für die Festlegung der Eckpunkte des empirischen Forschungsdesigns und die Konzipierung eines Interviewleitfadens für ein qualitatives Befragungssetting.

\subsubsection{Methodisches Vorgehen}

Im Rahmen der Literaturanalyse wurde zunächst gemäß der „Grounded Theory“ ein offenes Herangehen an die Thematik gewählt, um möglichst alle Aspekte abzudecken (Akremi 2014), was sich in der thematischen Bandbreite der Leitthesen niedergeschlagen hat. Vor diesem Hintergrund bot sich ein qualitativer empirischer Forschungsansatz mit Befragung von Expertinnen und Experten in Schweizer Unternehmen und Verwaltungsorganisationen auf Grundlage eines Interviewleitfadens als Primärerhebung an. Die Auswahl der Interviewteilnehmenden erfolgte mittels einer Quotenauswahl (Akremi 2014) mit Fokus auf die Unternehmensbereiche Human Resource Management und Informationstechnologie (IT). Hinsichtlich der Rahmensetzungen für Big Data wurden ergänzend Expertinnen und Experten mit einem Erfahrungshintergrund in den Bereichen Datenschutz und Gewerkschaften bei den Interviews berücksichtigt. Zielsetzung war die Abdeckung dreier Befragungsperspektiven. Bei der IT-Perspektive ging es darum, einen Einblick darüber zu bekommen, wie der derzeitige technische Status quo in Bezug auf Big Data und deren Anwendung in den Unternehmen ist, welche Entwicklungen für die Zukunft zu erwarten sind und welche Grenzen sich hierbei abzeichnen. Bei der HR-Perspektive wurden analoge Fragestellungen herangezogen, allerdings mit Fokus auf den Entwicklungsstand, die Anwendungsbezüge und Potenziale im HRM. Die Interviewpartnerinnen und -partner der Rahmenrerspektive schließlich decken grundsätzliche Big-Data-Aspekte ab, die über die Unternehmensgrenzen hinausgehen. Insgesamt wurden im Befragungssample 15 Personen aus 13 Unternehmen und Organisationen befragt. Bei den Unternehmen handelt es sich primär um IT-, Dienstleistungs- unter Beratungsunternehmen mit Bezügen zur Thematik (Schweizerische Post, Swisscom, Deloitte, PwC, HRSC, foryouandyourcustomers, 
IBM). Weiterhin wurden Expertinnen und Experten aus der öffentlichen Verwaltung (Bund), dem gewerkschaftlichen Umfeld (Syndicom, Unia), dem Bildungssektor (Zürcher Hochschule für Angewandte Wissenschaften) und im Verbandswesen (Digital Switzerland) befragt. Von den 15 Expertinnen und Experten befassen sich 3 auf der Grundsatzebene möglicher Konsequenzen in der Außensicht mit der Thematik. 6 Personen verfügen über übergreifendes Fachwissen im unternehmensinternen Umfeld der Thematik und weitere 6 Personen sind in einzelnen Phasen des Einsatzes und der Nutzung von Big Data aktiv involviert, sodass eine große Bandbreite unterschiedlicher Betrachtungsstandpunkte in die Erhebung einfließen konnte (vgl. dazu Abb. 11.2).

Die Befragungen wurden im Frühjahr 2018 in Form von ca. einstündigen mündlichen Interviews durchgeführt. Sämtliche Interviews erfolgten unter Heranziehung eines semistrukturierten offenen Interviewleitfadens, der an den Leitthesen ansetzt und in einem Pretest überprüft wurde. Die offene Ausrichtung ermöglichte ein flexibles Eingehen auf die jeweilige Gesprächssituation (Helfferich 2014). Je nach Befragungsperspektive bzw. Kategorie der Befragten wurde demgemäß der Fragebogen vor dem jeweiligen Interview entsprechend modifiziert, ohne aber die Grundsystematik zu beeinträchtigen. Alle Interviews wurden aufgezeichnet und gemäß der Transkriptionsregeln von Kuckartz und Rädiker (2014) transkribiert. Die Transkripte wurden mit der Analysesoftware Atlas.ti erstellt und anschließend themenbezogen codiert und ausgewertet.

\subsubsection{Ergebnisse}

Im Verlauf der Interviews zeichnete sich schon bald ein wiederkehrendes Bild zum Vorgehen der in der Praxis angewandten Big-Data-Analysen ab, das in Abb. 11.3 dargestellt ist. Das Modell beschreibt prozessbezogen den Ablauf der Big-Data-Analyse im HRM vom Urheber der Daten bis hin zum Umgang der Unternehmen mit den daraus gewonnenen Informationen. Es gibt zwei Arten von auswertbaren Informationen: Zum einen verfügt das Unternehmen über interne Daten seiner Mitarbeitenden, welche im Rahmen des

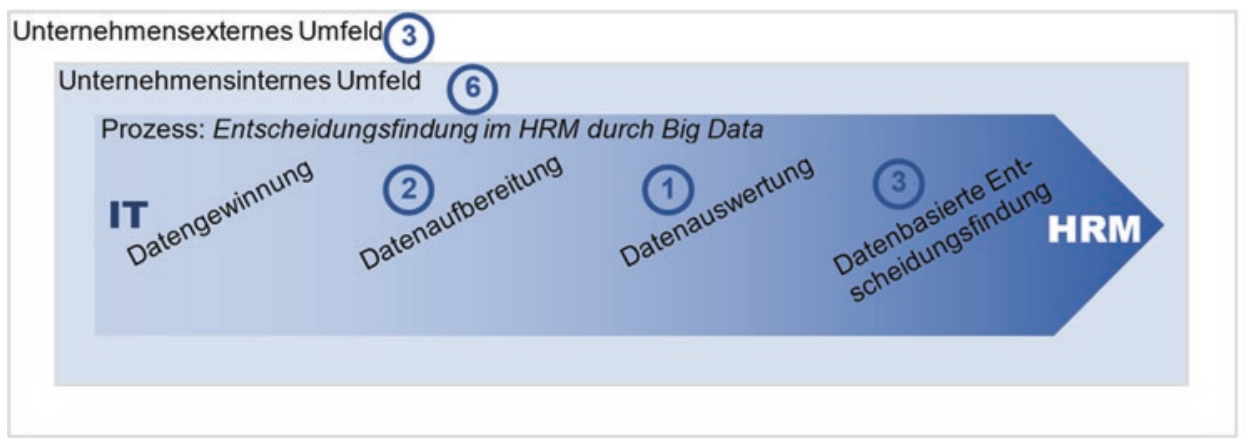

Abb. 11.2 Betrachtungsstandpunkte der Interviewpartnerinnen und -partner. (Quelle: eigene Darstellung. Die Zahlenkreise stehen für die Anzahl der Interviewteilnehmenden je Standpunkt) 


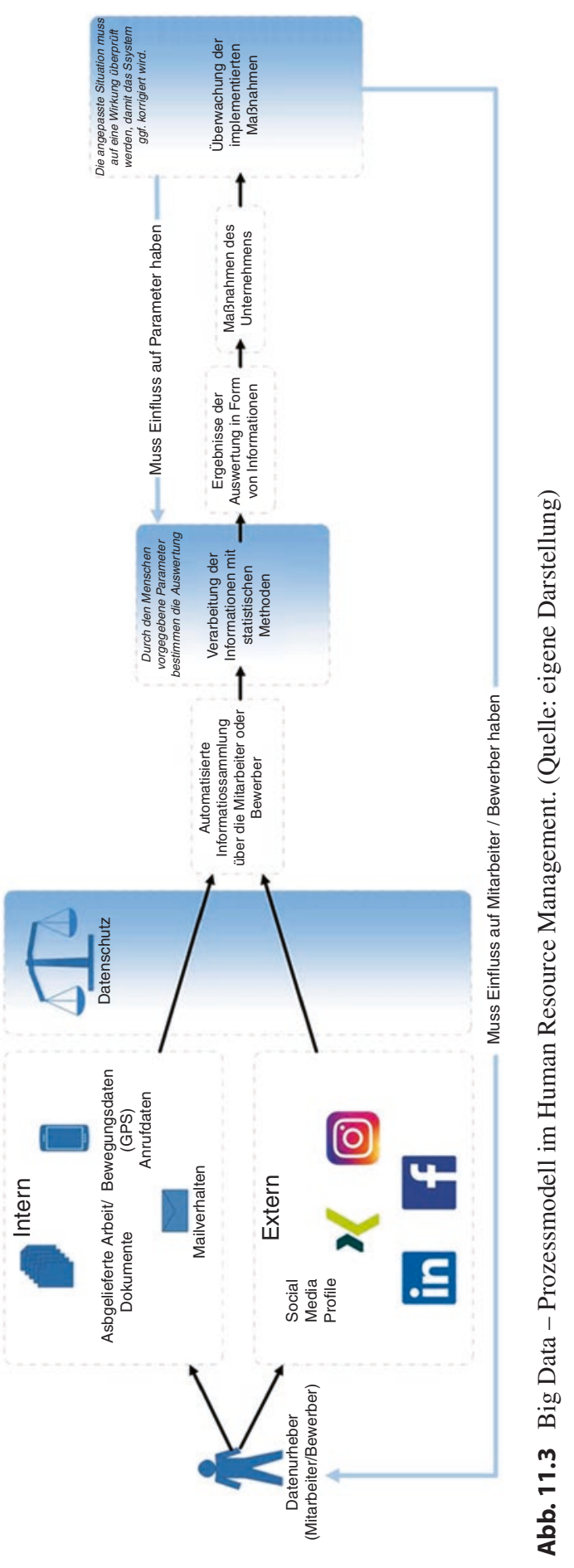


Arbeitsverhältnisses anfallen. Zudem gibt es externe Daten, welche öffentlich über Personen zugänglich sind. Bevor diese Daten in die Analyse einfließen können, müssen datenschutzrechtliche Aspekte berücksichtigt werden. Die danach verbleibenden Informationen können aufbereitet werden, sodass mit statistischen Analysemethoden Erkenntnisse gewonnen werden können. Zur Reduzierung von subjektiven Fehlerquellen sind die gesetzten Analyseparameter kontinuierlich zu überprüfen. Mit der Ausgabe der Ergebnisse ist der technische Teil der Big-Data-Analyse abgeschlossen. Es folgt nach einer Analyse und Interpretation der Resultate die Ableitung, Umsetzung und Wirkungsüberprüfung von HR-Maßnahmen. Nur bei einem kontrollierten Regelkreis kann festgestellt werden, ob anhand der Big-Data-Analysen korrekte Aussagen abgeleitet wurden. Mit dem Wissen aus dem Monitoring können nun die Methode bzw. die dahinter stehenden Algorithmen und die zugehörigen Parameter korrigiert werden, um die Genauigkeit der Analyse kontinuierlich zu verbessern.

Die Ergebnisse der qualitativen Analyse werden nachfolgend summarisch und hochaggregiert im Kontext der jeweiligen Leitthese dargelegt.

Leitthese 1: Unternehmen verfügen nicht über die nötigen Fachkräfte, um Big Data im HRM adäquat anwenden zu können.

Insgesamt wurde die Vermutung eines Big-Data-Fachkräftemangel, insbesondere auch mit spezifischem HR-Bezug, von den Befragungsteilnehmenden bestätigt. Es hat sich dabei gezeigt, dass das Rollenverständnis und Anforderungsprofil solcher HR-Big-DataExpertinnen und -experten noch sehr heterogen wahrgenommen wird. Einerseits werden hierfür vor allem neue HR-Expertinnen und -experten mit spezifischem Big-Data-Knowhow als sinnvoll erachtet, andere Befragte sehen im Gegensatz hierzu aber eher neue Schnittstellenfunktionen, die sich zwischen der IT und den anderen betrieblichen Funktionsbereichen, hier also dem HRM, herauskristallisieren. Weitgehende Einigkeit besteht allerdings über das erforderliche Kompetenzprofil von Big-Data-Expertinnen und -experten im HRM-Kontext: Voraussetzung sind umfassende Kenntnisse in Mathematik und Statistik, IT-Grundkenntnisse und Programmiererfahrung. Wichtig sind ferner Kenntnisse über die Gewinnung und Bereinigung von Daten. Zudem besteht die Notwendigkeit, als interdisziplinärer Brückenbauer dieses „Technik“-Wissen mit dem HR-Wissen zu verknüpfen und Ergebnisse den Stabs- und Linienfunktionen zu übermitteln. Die Expertinnen und Experten sollten ein Gespür für Problemlagen, Bedürfnisse und Potenziale der Linie in Bezug auf Personalthemen mitbringen. Darüber hinaus wurden auch Visualisierungsskills, User-Experience-Know-how und Beratungsfähigkeiten als sehr hilfreich angesehen. Zusammenfassend handelt es sich also um ein sehr anspruchsvolles Anforderungsprofil für HR-Big-Data-Expertinnen und -Experten, was den festgestellten Verfügbarkeitsmangel am Arbeitsmarkt mit erklärt. Idealtypische Ausbildungskombinationen wären zum Beispiel eine HR-Ausbildung in Verbindung mit einem Wirtschaftsinformatikstudium, oder eine IT-Ausbildung in Verbindung mit einer betriebswirtschaftlichen Zusatzausbildung mit HR-Schwerpunkt. Tendenziell werden zukünftige funktionale Schnittstellenprofile als eher im IT-Bereich verankert gesehen. Im IT-Sektor dürften somit in der Zukunft die Stellenprofile im Big-Data-Kontext spezifischer werden, während IT- 
Know-how sich vermehrt aber auch in den betriebswirtschaftlichen Funktionen zur Grundlagenkompetenz entwickelt. HR-Businesspartner werden aus Sicht der Befragten zukünftig auch in der Lage sein müssen, mit HR-Big-Data-Expertinnen und -Experten der IT gemeinsam an der Ableitung von personalen Handlungsstrategien und -maßnahmen zu arbeiten. Großunternehmen dürften es beim Neuaufbau entsprechender Analysekompetenzen im IT- und HR-Bereich ressourcenbedingt deutlich leichter haben. Entsprechende Profile finden sich derzeit wohl deshalb vor allem bei ihnen. Für die Zukunft scheint ein erheblicher Big-Data-Qualifizierungsbedarf für IT- und HR-Expertinnen und -Experten wahrscheinlich, der auch für mittelständische Unternehmen zum Tragen kommt, die ihre Organisationsintelligenz traditionell stark über die fachliche Weiterqualifizierung ihrer Mitarbeitenden weiterentwickeln.

Leitthese 2: Es werden primär Stammdaten für HR-Analytics genutzt. Big Data gemäß der gängigen weitaus breiter gefassten Definition kommt nicht oder nur in geringem Umfang zum Einsatz, da die derzeit genutzten Daten mengenmäßig überschaubar sowie statisch und semi-strukturiert sind.

Bei der Durchführung der Interviews hat sich gezeigt, dass ein sehr heterogenes Verständnis von Big Data bei den befragten Praktikern vorherrscht, sodass im Interview wiederholt erst eine begriffliche Klärung im Sinne des theoretisch-konzeptionellen Verständnisses erforderlich war. Auch wenn keine allgemein akzeptierte Definition von Big Data vorgefunden wurde, so erfolgten die Beschreibungen des dahinter vermuteten Sachverhalts doch immer mittels ähnlicher Aspekte rund um die drei V (Volume, Variety und Velocity), die aber explizit so nicht bei allen Befragungsteilnehmenden bekannt waren. Es wurde in allen Interviews von der Analyse einer großen, unstrukturierten Datenmenge gesprochen, mit dem Ziel, durch Datenverknüpfung Erkenntnisse zu gewinnen. Es wurde ferner bestätigt, dass dort, wo bis heute umfangreichere Auswertungen mit HR-Daten gemacht werden, es sich nicht um Big Data resp. People Analytics im engeren Sinne handelt, da die untersuchten Daten in der Regel weder unstrukturiert sind noch ein Big-DataDatenvolumen repräsentieren. Offenbar werden in den untersuchten Unternehmen und Organisationen überwiegend auch keine Daten verarbeitet, welche laufend von den Mitarbeitenden selbst generiert werden (Bewegungsdaten), sondern primär Personalstammdaten (Geburtsdatum, Name, Geschlecht, Ziele, etc.). In einigen Unternehmen werden aber die bisherigen Analysen zunehmend in Richtung des theoretisch-konzeptionellen Begriffsverständnisses von Big Data/People Analytics erweitert.

Leitthesen 3 und 10: Schweizer Unternehmen scheuen den Einsatz von Big Data aus Reputationsgründen. Die negative Beeinflussung der öffentlichen Wahrnehmung von Big Data durch die Medien führt zu einer verringerten Akzeptanz von Big Data in der Gesellschaft.

Aufgrund des engen Zusammenhangs von These 3 und 10, der sich auch in den geführten Gesprächen gezeigt hat, werden beide Aspekte in einer übergreifenden Ergebnisdarstellung integriert betrachtet. Alle Teilnehmenden wurden nach ihrer Wahrnehmung von der Aufgeklärtheit und der Beeinflussbarkeit der Schweizer Bevölkerung in Bezug auf Big Data befragt. Diesbezüglich hat sich kein einheitliches Bild ergeben und die Einschätzun- 
gen deckten die gesamte Bandbreite an Möglichkeiten ab. Die Expertinnen und Experten sind sich allerdings weitestgehend einig darüber, dass das Bild, welches von den Medien rund um die Thematik „Big Data“ vermittelt wird, eher negativ behaftet ist und dass immer wiederkehrende negative Beispiele von Datenmissbrauch die Skepsis und Sensibilität innerhalb der Bevölkerung eher fördern. Eine höhere Akzeptanz könnte man aus Sicht der Befragten erreichen, wenn die Betroffenen besser und sachlich über Big Data informiert werden und auch für sich einen persönlichen Mehrwert wahrnehmen. Dies würde eine entsprechende Datennutzung durch Unternehmen im Sinne einer bewussten und freiwilligen Datenbereitstellung der Mitarbeitenden als Beitrag zur Unternehmensentwicklung, aber eben auch für die persönliche Weiterentwicklung legitimieren. Das individuelle Nutzenargument scheint insofern, kontrastierend zur Bedrohung eines Missbrauchs der eigenen Personendaten, zusammen mit einem Grundvertrauen in die Seriosität und Wertstiftung der Analysen für alle essenziell für eine Akzeptanz durch die Mitarbeitenden. Eine reputationsbedingte Zurückhaltung von Big Data wurde von den Befragten nicht generell bestätigt. Thematisiert wurde aber, dass einige Unternehmen im Zuge von bekannten Datenskandalen bezüglich Datenanalysen eher zurückhaltender kommunizieren. Die Gefahr von Reputationsschäden wird also durchaus gesehen und auch ein damit verbundener zusätzlicher Anreiz, sich strikt an die gesetzlichen Rahmenvorgaben in Bezug auf die Nutzung von Personaldaten zu halten. Es wurde ferner auch von einigen Befragten darauf hingewiesen, dass medial eher negativ belegte technische Begriffe wie „Big Data“ oder „Datenanalyse“ in der Innen- und Außenkommunikation eventuell durch alternative synonyme Begrifflichkeiten ersetzt werden sollten.

Leitthese 4: Big Data im HRM wird in Zukunft die Rekrutierung vollautomatisieren und Rekrutiererinnen und Rektrutierer sowie und Pesonalvermittlerinnen und -vermittler größtenteils ersetzen.

Die Feststellung, wonach Big Data im HRM in den Unternehmen und Organisationen noch sehr unterschiedlich (nicht) angekommen ist, spiegelt sich auch in den Aussagen in Bezug auf die Personalbeschaffung wider. Man ist sich eines möglichen Einsatzpotenzials durchaus bewusst, im Alltag ist aber eine Nutzung dieser Potenziale vielfach noch nicht in der Praxis umgesetzt. Hinsichtlich der zukünftigen Entwicklung waren sich aber alle Befragten weitgehend einig darüber, dass Big Data vor allem bei der Auffindung von spezifischen Kandidierenden und Zielgruppen und zur Informationsanreicherung im Selektionsprozess genutzt werden kann. Eine Vollautomatisierung im Sinne eines weitgehenden Ausschlusses der menschlichen Dimension im Rekrutierungsverfahren wird aber, zumindest in einem absehbaren Zeithorizont, nicht erwartet. Auch in Zukunft werden in der Einschätzung der Befragten menschliche Interventionen und Entscheidungen im Rekrutierungs- und Selektionsverfahren einen wichtigen Stellenwert einnehmen. Unterschiede gab es dabei allenfalls in der Einschätzung der phasenbezogenen Relevanz und des Ausmaßes menschlicher Inputs. So sind einige Befragte etwa der Meinung, dass die definitiven Personalentscheide auch in fernerer Zukunft immer noch durch Menschen getroffen werden, während andere glauben, dass die Algorithmen sich irgendwann über Selbstlernprozesse und menschliche Feedbackschlaufen so verbessern könnten, dass sie auch eines 
Tages diesen letzten Auswahlschritt für die am besten passenden Bewerbenden selber durchführen können. Die diesbezüglichen Skeptiker sehen vor allem Defizite der Maschinen bei der Wahrnehmung und Interpretation zwischenmenschlicher Aspekte, die für eine harmonische und erfolgreiche Zusammenarbeit in Teams essenziell sind. Insgesamt wird davon ausgegangen, dass es das Arbeitsprofil des Recruiters auch in Zukunft noch geben wird, wobei jedoch der Einsatz unterstützender technischer Hilfsmittel und Algorithmen deutlich zunehmen wird. Durch die vermuteten Effizienzsteigerungen könnte sich die Anzahl der Recruiter und Personalvermittler im traditionellen Sinne aber eventuell reduzieren, vor allem auch in Bezug auf die Vermittlung temporärer Stellen, die vermutlich eher stärker automatisierbar sein wird.

Leitthese 5: Unternehmen wissen in Zukunft über potenzielle individuelle Veränderungen (z. B. potenzielle Kündigung oder Krankheit) ihrer Mitarbeitenden deutlich besser Bescheid.

Geplante und ungeplante berufliche Entwicklungsschritte von Mitarbeitenden sowie Bewerbenden sind bei Arbeitgeberinnen und Arbeitgebern häufig von großem Interesse. So könnte etwa eine Früherkennung von potenziellen krankheits- oder kündigungsbedingten Personalausfällen und -engpässen eine interessante Zielsetzung von Big-DataAnalysen sein. Die befragten Personen sehen hier durchaus Unterstützungsmöglichkeiten für die HR-Berater, vor allem aber für die Linienführungskräfte, insbesondere, wenn diese eine hohe Führungsspanne zu bewältigen haben und kritische Entwicklungen leichter übersehen oder zu spät wahrgenommen werden können. Von einigen Befragten wurde allerdings in Verbindung mit bestehenden Arbeitsverhältnissen kritisch angemerkt, dass es an sich eine Kernaufgabe der Führungsarbeit von Vorgesetzten sei, über die Motivationslagen, beruflichen Absichten und gesundheitliche Einschränkungen oder Veränderungen ihrer Mitarbeitenden informiert zu sein. Eine Datenanalyse macht, wie in anderen Bereichen auch, aber hier nur Sinn, wenn identifizierte Problembereiche tatsächlich dann auch zur Initiierung von Maßnahmen führen und nicht einfach neue Datenfriedhöfe generiert werden. Befragte mit einer eher kritischen Grundhaltung zur Big-Data-Anwendung in diesem Bereich bezweifeln auch die derzeitige Möglichkeit zur Entwicklung von Algorithmen, die weiche Faktoren in hinreichendem Masse in die Analyse einfließen lassen. Entwicklungs- und Verhaltensprognosen mit einem Früherkennungsanspruch für das Personal in diesen Bereichen setzen nämlich hoch entwickelte technische Systeme voraus, die mit flexiblen Parametern und Systemvorgaben arbeiten und maschinell lernen, also über künstliche Intelligenz verfügen. Eine hohe Gefahr bestehe darin, dass nicht oder unzureichend lernfähige Systeme, die initial fehlerhaft aufgesetzt wurden, immer fehlerhafte Analyseresultate liefern und die Qualität der Entscheidungen nicht verbessern werden. In diesem besonders heiklen Themenbereich der Verhaltensprognose für das potenzielle und aktuelle Personal bezüglich grundlegender individueller Entwicklungen und Entscheidungen sind gemäß der interviewten Expertinnen und Experten die rechtlichen Rahmensetzungen des Datenschutzgesetzes und des Arbeitnehmerschutzgesetzes von besonderer Bedeutung. Analysen, die Rückschlüsse auf einzelne Personen erlauben, sind durch diese Gesetze derzeit untersagt, sodass nur aggregierte und anonymisierte Aussagen auf Unter- 
nehmens-, Bereichs- oder gegebenenfalls auch Teamebene aus den Big Data abgeleitet werden dürften. Andernfalls müsste eine explizite Einwilligung zur Verwendung individueller Daten und zur Ableitung individualisierter Aussagen und Maßnahmen von betroffenen Mitarbeitenden eingeholt werden. Anwendungsbezüge gibt es hier also primär im Kontext der Beurteilung größerer Mitarbeitendeneinheiten.

Leitthese 6: Dank der Anwendung von HR-Analytics können Mitarbeitende in Zukunft anhand ihrer Leistungsprofile und Stärken besser eingesetzt und gefördert werden.

Bei den Befragten zeigte sich in Bezug auf performancebezogene Big-Data-Analysen eher eine skeptische Potenzialeinschätzung mit einer zur These 5 ähnlichen Argumentationsrichtung. Lediglich ein Befragungsteilnehmer hat sich diesbezüglich klar positiv geäußert. Wie bei These 5 wird auch in diesem Zusammenhang eine Abgrenzung von Überwachungs- und Hilfestellungsperspektive als nur schwer möglich angesehen. Nutzungsmöglichkeiten scheinen primär zur Entscheidungsunterstützung auf aggregierter Ebene gegeben zu sein. Die Bewertung von Leistungsprofil, der richtigen beruflichen Positionierung und die Mitarbeitendenförderung werden von den meisten Befragten auch als zentrale Führungsaufgabe der Leitungspersonen gesehen. Das Missbrauchsrisiko entsprechender Big-Data-Analysen in ethisch bedenklichen eventuellen gesetzlichen Grauzonen, oder gar widerrechtlich, wird von den Befragungsteilnehmenden als problematisch angesehen.

Leitthese 7: Dank der Erkenntnisse von HR-Analytics gewinnen die Unternehmen Wissen, das es Ihnen ermöglicht, die Mitarbeitenden zu motivieren.

Diese Leitthese weist ebenfalls Überschneidungen zu den Thesen 5 und 6 auf und die Argumentationsführungen der Befragten sind vergleichbar: Die Motivation der Mitarbeitenden ist eine Führungsaufgabe und HR-Analytics-Systemlösungen bieten allenfalls unterstützende Hilfestellungen auf aggregierter Ebene im Sinne von Mustererkennungen an. Es wird, sofern die technische und rechtliche Umsetzbarkeit gegeben ist, ein gewisses Unterstützungspotenzial von HR-Analytics für die Führungskräfte ausgemacht, auch wenn der entsprechende Bedarf derzeit wohl eher gering ist. Dies hängt auch damit zusammen, dass kollektive Mitarbeitendenbefragungen, die üblicherweise Motivationsaspekte dezidiert erheben, schon heute breit in den Unternehmen und der öffentlichen Verwaltung etabliert sind. In einer verstetigten Form bieten einige Softwaresysteme bereits heute, unabhängig von einem Big-Data-Kontext, zyklische Befragungssettings und automatisierte Standardauswertungen an.

Leitthese 8: Im „War for Talents" haben Unternehmen, die Big Data in der Rekrutierung einsetzen, einen Wettbewerbsvorteil.

Insbesondere im IT-Bereich, aber auch in anderen Spezialfunktionen wird seitens der Befragten ein intensiver Wettbewerb um qualifizierte Fach- und Führungskräfte bestätigt. Für eine Anwendung von Big-Data-Analysen wird hier sowohl im internen, wie im externen Arbeitsmarkt ein gegebenes Erfolgspotenzial bestätigt. Besonders für die interne Identifikation von Talenten, und damit auch deren Weiterentwicklung und Bindung an das Unternehmen, sehen die Expertinnen und Experten kostenreduzierende und qualitätserhöhende Einsatzmöglichkeiten von Big-Data-Anwendungen, die auch im gegebenen Rechts- 
rahmen und bei gegebener Datenlage denkbar sind, was bei der Identifikation externer Talente weniger gewährleistet ist.

Leitthese 9: Durch die bislang restriktive Gesetzgebung wird der Fortschritt der Anwendung von Big Data im HRM gehemmt.

Zur Nutzung von Personendaten müssen die Dateneigner Rechte an ihren Daten abtreten oder Verwendungsrechte gewähren. Der aktuell gegebene Schweizer und EURechtsrahmen sieht vor, dass der gesamte Prozess der Sammlung, Auswertung und Lagerung von Daten vollständig und transparent dargelegt werden muss. Zudem muss das datennutzende Unternehmen jederzeit die Daten auf Wunsch des Datenurhebers löschen können und diesen Vorgang ebenfalls nachweisen. Bei zweckgebundenen Datenerhebungen muss der Einsatzzweck deklariert werden und die Mitarbeitenden müssen dem Zweck explizit zustimmen. Diese derzeit gegebenen gesetzlichen Hürden werden von etwa einem Drittel der Befragten als angemessen und auch erforderlich betrachtet. Mit der Einführung der EU-Datenschutzgrundverordnung sei der Datenschutz nicht unbedingt strenger geworden, aber die Unternehmen müssten anspruchsvolleren Nachweispflichten nachkommen. Dass der bisherige und sich abzeichnende zukünftige Rechtsrahmen insofern einschränkt, dass nicht alles gemacht werden darf, was gemacht werden könnte, wird von allen Expertinnen und Experten bestätigt. Die meisten Befragten rechnen für die Zukunft aber nicht mit einer weiteren Verschärfung des Datenschutzes. Im Falle einer Lockerung der Rechtssetzung, die von den Befragten als eher unwahrscheinlich betrachtet wird, gäbe es wohl ein deutliches Mehr an potenziellen Big-Data-Anwendungsfeldern im HRBereich, aber untrennbar auch ein zusätzliches Überwachungsrisiko aus Sicht der Mitarbeitenden.

Losgelöst von der Behandlung der Einzelthesen aus Sicht der Befragungsteilnehmenden kann übergreifend festgehalten werden, dass sich alle Expertinnen und Experten einig sind, dass der Umgang mit und die Nutzung von Big Data als Teilaspekt der Digitalisierung in der Unternehmenswelt ein Thema bleiben wird. Das spezifische Potenzial von Big Data für das HRM wird jedoch differenzierter gesehen, und hier gibt es noch größere Unsicherheiten bezüglich der weiteren Entwicklung und potenzieller Chancen und Risiken. Die ökonomische Sinnhaftigkeit einzelner Einsatzbereiche von Big Data muss sich aus Sicht der Befragten langfristig erst noch bestätigen. Zumindest einzelne Big-DataAnalysefelder zur Unterstützung der Entscheidungsfindung im gegebenen Rechtsrahmen dürften wohl in der Zukunft auch im HRM mit hoher Wahrscheinlichkeit bearbeitet werden.

\subsection{Fazit}

Ist Big Data ein relevanter Trend für das Human Resource Management in Schweizer Unternehmen? Auch wenn es kein starkes „Ja“ ist, so kann man die Frage gleichwohl auf Basis der erfolgten konzeptionellen und leitthesenorientierten empirischen Analyse bejahen. Eine datenbasierte Entscheidungsfindung ist zunehmend auch ein Thema in den Personalabteilungen Schweizer Unternehmen. Allerdings heißt „Datenanalyse“ nicht zwin- 
gend auch die Nutzung und Analyse von „Big Data“. Was Letzteres ist, und welche Analysemethoden hiermit verbunden sind, wird in der Praxis noch sehr heterogen wahrgenommen. Vielfach wird mit Big Data oder Data Analytics im HR-Bereich einfach die Analyse von Personalstammdaten oder ein Einsatz von Filtern z. B. beim Scanning von Lebensläufen gleichgesetzt. Ein einheitliches Verständnis dessen, was Big Data ist und was Big Data für den HR-Bereich heißt, scheint derzeit noch nicht zu existieren. Seitens der Befragten werden datenbedingt Anwendungspotenziale aktuell vor allem im Bereich des Gesundheitsmanagements und der Rekrutierung gesehen. Im Kontext der Rekrutierungsprozesse scheint die Entwicklung bislang am weitesten vorangeschritten zu sein, und Weiterentwicklungen sind hier wohl auch am wahrscheinlichsten, z. B. in Form von der Einbettung automatisierter Videoanalysen bei virtuellen Vorstellungsgesprächen.

Obschon technisch schon Vieles möglich wäre, hindern unter anderem rechtliche Restriktionen und auch ethische Bedenken die Entscheidungsträgerinnen und -träger im HRM derzeit noch daran, das volle Potenzial von Big Data auszunutzen. Die Handhabung von Mitarbeitendendaten gilt als hochsensibel. Ohne die Zustimmung der betroffenen Personen dürfen Unternehmen nicht ungehindert deren Daten sammeln und analysieren. Der Gesetzgeber und auch die Gewerkschaften in der Schweiz sind diesbezüglich sehr sensibilisiert. Darüber hinaus droht im Falle eines fahrlässigen Umgangs mit Personaldaten und bei einem Datenmissbrauch ein hohes Reputationsrisiko für Unternehmen und andere Organisationen.

Das Nutzungspotenzial von Big Data scheint in anderen Funktions- und Analysebereichen wie etwa im Marketing oder in der Logistik ausgeprägter zu sein als im HRM. Derzeit werden im HR-Bereich in den untersuchten Unternehmen und Organisationen eher experimentell und testweise Big-Data-Analysen im engeren Sinne vorgenommen. Von einer grundlegenden Etablierung in der Praxis kann vor diesem Hintergrund demnach noch nicht gesprochen werden. Die ökonomische Vorteilhaftigkeit und der Nutzen für die Mitarbeitenden müssen sich in den nächsten Jahren erst noch weiter konkretisieren. Wichtig dabei scheint, dass betroffenen Personen Big-Data-Analyseprozesse und die Ableitung von Maßnahmen transparent gemacht werden und dass auch Mitarbeitende einen klaren Mehrwert für sich selbst und das Unternehmen erkennen können. Dann wird die Nutzung von Big Data wohl auch im HRM der Zukunft irgendwann zu einer Selbstverständlichkeit werden.

\section{Literatur}

Akremi, L. (2014). Stichprobenziehung in der qualitativen Sozialforschung. In N. Bauer \& J. Blasius (Hrsg.), Handbuch Methoden der empirischen Sozialforschung (S. 265-282). Wiesbaden: Springer.

Almog, G. (2018). Traditional recruiting isn't enough: How AI is changing the rules in the human capital market. https://www.forbes.com/sites/groupthink/2018/02/09/traditional-recruiting-isntenough-how-ai-is-changing-the-rules-in-the-human-capital-market/\#170ac52c274a. Zugegriffen am 09.02.2018. 
Barman, A. (2015). Big data in human resource management - Developing research context. Assam: Assam University.

Beck, M., Groggert, S., \& Kühn, A. (2016). So geht Digitalisierung - Erfolgsgeschichten aus dem Mittelstand. Mühlheim an der Ruhr: EffizienzCluster Management GmbH.

Bendel, O. (2018a). Big Data. https://wirtschaftslexikon.gabler.de/definition/big-data-54101/version-277155. Zugegriffen am 19.02.2018.

Bendel, O. (2018b). Digitalisierung. https://wirtschaftslexikon.gabler.de/definition/digitalisierung-54195/version-277247. Zugegriffen am 19.02.2018.

Berendes, K., Kumpf, J., \& Delarue, M. (2016). Strategische Personalplanung und HR Analytics Navigationshilfe für das Management am Beispiel der AOK Hessen. HMD Praxis der Wirtschaftsinformatik, 53, 828-837.

Boorman, C. (2017). Beispiele für das Internet of Things. https://automic.com/de/blog/10-beispielef-r-das-internet-things-iot. Zugegriffen am 27.01.2017.

Bundesamt für Justiz BJ. (2017). Stärkung des Datenschutzes. https://www.bj.admin.ch/bj/de/home/ staat/gesetzgebung/datenschutzstaerkung.html. Zugegriffen am 15.09.2017.

Bundesamt für Kommunikation (BAKOM). (2018). Strategie Digitale Schweiz. Bern: BAKOM/GDS.

Columbus, L. (2016). Ten Ways Big Data Is Revolutionizing Marketing And Sales. https://www. forbes.com/sites/louiscolumbus/2016/05/09/ten-ways-big-data-is-revolutionizing-marketingand-sales/\#5a91c6fc21cf. Zugegriffen am 13.06.2020.

Dietzfelbinger, M., Mehlhorn, K., \& Sanders, P. (2014). Algorithmen und Datenstrukturen. Berlin: Springer.

Dörfel, L. (2013). Instrumente und Techniken der Internen Kommunikation. Berlin: scm.

Ebert, C. (2017). Powerful ways HR can leverage Big Data. http://www.digitalistmag.com/futureof-work/2017/08/31/5-powerful-ways-hr-can-leverage-big-data-05242673. Zugegriffen am 10.03.2018.

Eichhorst, W., Hinte, H., Spermann, A., \& Zimmermann, K. F. (2015). Die neue Beweglichkeit: Die Gewerkschaften in der digitalen Arbeitswelt. Bonn: Institute of Labor Economics IZA.

Eidgenössisches Personalamt (EPA). (2019). Personalstrategie Bundesverwaltung 2020-2023. Bern: BBL.

Gehrmann, M. (2014). Was man über den USA Patriot Act wissen sollte. www.pretiose-blog.com: https://pretioso-blog.com/was-man-ueber-den-usa-patriot-act-wissen-sollte/\#. Wu7PpR6sYzN. Zugegriffen am 28.03.2018.

German Research Center for Artificial Intelligence. (2016). Künstliche Intelligenz: Overhyped oder unterschätzt? CeBIT future talkYouTube. https://www.youtube.com/watch?v=77QhkWNOqS8. Zugegriffen am 13.06.2020.

Gewirtz, D. (2018). Velocity and variety. Understanding the three vs of big data. https://www.zdnet. com/article/volume-velocity-and-variety-understanding-the-three-vs-of-big-data/. Zugegriffen am 21.03.2018.

Greif, H., Kühnis, N., \& Warnking, P. (2016). Digitalisierung - Wo stehen Schweizer KMU? Zürich: PwC Schweiz.

Helfferich, C. (2014). Leitfaden- und Experteninterviews. In N. Baur \& J. Blasius (Hrsg.), Handbuch Methoden der empirischen Sozialforschung (S. 559-574). Wiesbaden: Springer.

Hofmann, S., \& Meyer, M. A. (2017). Datenschutz in der Schweiz. Expert Focus, 12(06), 2017.

Hunkeler, V. (2019). Rekrutierung digital denken. https://intranet.bfh.ch/BFH/Documents/Dienste/ Rechtsdienst/Rechtliche_Grundlagen/Studium/FBW/2008\%2006\%2023\%20ZulR\%20 MA\%20BA.pdf. Zugegriffen am 07.01.2019.

Institut für angewandte Arbeitswissenschaft (Hrsg.). (2016). Digitalisierung und Industrie 4.0. Düsseldorf: Institut für angewandte Arbeitswissenschaften.

Kappes, C. (2016). Künstliche Intelligenz. Grenzen und Kritik. https://merton-magazin.de/ k\%C3\%BCnstliche-intelligenz-\%E2\%80\%93-grenzen-und-kritik Zugegriffen am 04.04.2018. 
Kefron Limited (Hrsg.). (2012). The paperless office - fact or fiction? Dublin: Kefron Limited.

Köpke, S. (2020). Personalentwicklung im digitalen Wandel: 8 gute Gründe für eine Digitalisierung der Personalentwicklung. https://www.babbelfuerunternehmen.de/blogs/de/personalentwicklungim-digitalen-wandel. Zugegriffen am 12.06.2020.

Kroker, M. (2018). Weltweite Datenmengen sollen bis 2025 auf 175 Zetabytes wachsen -8 mal so viel wie 2017. https://blog.wiwo.de/look-at-it/2018/11/27/weltweite-datenmengen-sollen-bis-2025auf-175-zetabyte-wachsen-8-mal-so-viel-wie-2017/\#: :text=Vor\%20allem\%20das\%20Datenvolumen\%20in,beschleunigt\%20sich\%20sogar\%20eher\%20noch. Zugegriffen am 11.06.2020.

Kuckartz, U., \& Rädiker, S. (2014). Datenaufbereitung und Datenbereinigung in der qualitativen Sozialforschung. In N. Bauer \& J. Blasius (Hrsg.), Handbuch Methoden der empirischen Sozialforschung (S. 383-396). Wiesbaden: Springer.

Lampart, D., \& Cirigliano, L. (2017). Digitalisierung muss den Berufstätigen nützen: Analyse und Handlungsbedarf. Bern: Schweizerischer Gewerkschaftsbund.

Lindner, R. (2018). Frau stirbt nach Unfall mit selbstfahrendem Auto von Uber. Frankfurter Allgemeine. http://www.faz.net/aktuell/wirtschaft/diginomics/frau-stirbt-nach-unfall-mit-selbstfahrendem-autovon-uber-15502443.html. Zugegriffen am 19.03.2018.

Liniger, R. (2015). Schwache Arbeitnehmerverbände - große Ungleichheit. https://apunto-online. ch/arbeitswelt/showData/schwache-arbeitnehmerverbaende-grosse-ungleichheit. Zugegriffen am 15.04.2018.

Meier, C., \& Franke, R. (2016). Aus- und Weiterbildung: Alles wird digital. Wirtschaft\&Weiterbildung, Oktober, S. 30-33.

Miller, R. (2015). Inside Facebook's Blu-ray Cold Storage Data Center. https://datacenterfrontier. com/inside-facebooks-blu-ray-cold-storage-data-center/. Zugegriffen am 05. 05.2018.

Minnich, S. (2020). Die Vorteile und Nachteile des Cloud Computing. https://www.heise.de/download/blog/Die-Vorteile-und-Nachteile-des-Cloud-Computing-3713041. Angefragt Zugegriffen am 10.06.2020.

Moeser, J. (2017). Starke KI - schwache KI. Was kann Künstliche Intelligenz? https://jaai.de/starkeki-schwache-ki-was-kann-kuenstliche-intelligenz-261/. Zugegriffen am 12.06.2020.

Müller, N., \& Kulemann, P. (2017). Digitalisierung und Arbeitsqualität: Eine Sonderauswertung auf Basis des DGB-Index Gute Arbeit 2016 für den Dienstleistungssektor. Berlin: ver.di - Vereinigte Dienstleistungsgewerkschaft.

O'Reilly, M. (2012). Volume - Velocity - Variety. What you need to know about Big Data. https:// www.forbes.com/sites/oreillymedia/2012/01/19/volume-velocity-variety-what-you-need-toknow-about-big-data/2/\#165d798370a1. Zugegriffen am 10.06.2018.

Pardini, G., Hügli, D., \& Schori, F. (2017). Gute Arbeit in der digitalen Schweiz. Bern: Gewerkschaft syndicom.

Plattner, H. (2017). Big Data. http://www.enzyklopaedie-der-wirtschaftsinformatik.de/lexikon/ daten-wissen/Datenmanagement/Datenmanagement\%2D\%2DKonzepte-des/Big-Data. Zugegriffen am 13.06.2020.

Richards, N. M., \& King, J. H. (2014). Big data ethics. Winston-Salem, USA. Wake Forest Law Review 393.

Schieb, J. (2017). Kritik an künstlicher Intelligenz nimmt zu. https://www.schieb.de/753933/kritikan-kuenstlicher-intelligenz-nimmt-zu. Zugegriffen am 13.06.2020.

Schneider-Ammann, J., Bossardt, M., Helbling, D., \& Mègret, D. (2017). Digitales Manifest. Zürich: digitalswitzerland.

Schröder, L. (2015). Wie sich die Digitalisierung entwickelt und warum die Zukunft in der Guten Arbeit liegt. Frankfurt a. M.: Campus.

Selke, S., Biniok, P., Achatz, J., \& Späh, E. (2018). Gutachten: Ethische Standards für Big Data und deren Begründung. Berlin: Bundesministerium für Bildung und Forschung.

Seufert, A. (2014). Entwicklungsstand, Potentiale und zukünftige Herausforderungen von Big Data Ergebnisse einer empirischen Studie. HMD Praxis der Wirtschaftsinformatik, 51, 412-423. 
Siemens AG (Hrsg.). (2013). Industrie 4.0 - Die vierte Industrielle Revolution. München: Siemens AG.

Siepermann, M. (2018). Internet der Dinge. https://wirtschaftslexikon.gabler.de/definition/internetder-dinge-53187/version-276282. Zugegriffen am 13.06.2020.

Smith, K. (2020). Facebook Nutzerzahlen. https://www.brandwatch.com/de/blog/facebookstatistiken/\#: :text=Es\%20wurden\%20mehr\%20als\%20250, entspricht\%20350\%20Millionen\%20Fotos\%20t\%C3\%A4glich abgerufen. Zugegriffen am 11.06.2020.

Stauffer, H. (2017). Wo Big Data heute genutzt wird. Business Intelligence topsoft Fachmagazin, $17(1), 28-31$.

Stutz, M. (2018). Auswirkungen der neuen EU DSGVO und des revidierten Schweizer DSG für Arbeitgeber. https://www.mme.ch/de/magazin/auswirkungen_der_neuen_eu_dsgvo_und_des_ revidierten_schweizer_dsg_fuer_arbeitgeber/.Zugegriffen am 04.04.2018.

Verordnung (EU) 2016/679 des Europäischen Parlaments und des Rates zum Schutz natürlicher Personen (2016). Brüssel: Europäische Union.

Widmer, U. (2016). Datenschutz: Was uns das EU-Recht angeht. Computerworld, S. 62-65.

Zwitter, A. (2014). Big data ethics. Big Data \& Society, 1(2), 1-6.

Reissich, Georg Konsekutives Masterstudium in Betriebswirtschaft an der Berner Fachhochschule mit Vertiefung in Corporate Business Development. Davor Bachelorstudium in Wirtschaftsingenieurwesen. Aktuell Leiter des Bereichs Qualität, Gesundheit, Sicherheit und Umwelt bei Blaser Swisslube AG.

Rohr, Geraldine Konsekutives Masterstudium in Betriebswirtschaft an der Berner Fachhochschule mit Vertiefung in Corporate Business Development. Davor Bachelorstudium in International Management. Aktuell Consultant für diverse Themen in der Schnittstelle zwischen Betriebswirtschaft und Technologie bei APP Unternehmensberatung AG.

Wanzenried, Bernadette Konsekutives Masterstudium in Betriebswirtschaft an der Berner Fachhochschule mit Vertiefung in Corporate Business Development. Davor Bachelorstudium in International Management. Parallel mehrere Tätigkeiten bei der öffentlichen Verwaltung, aktuell administrative Kursleitung bei der Wirtschaft und Kaderschule Bern.

Schellinger, Jochen Studiengangsleiter Master of Science in Business Administration an der Berner Fachhochschule Wirtschaft. Lehr-und Forschungstätigkeiten in den Bereichen Strategisches Management, Marketing und Personalmanagement. Davor 15 Jahre Praxistätigkeit bei der Deutschen Sparkassenorganisation, im Haniel-Konzern, an der Universität Tübingen sowie in der Daimler AG.

Open Access Dieses Kapitel wird unter der Creative Commons Namensnennung 4.0 International Lizenz (http://creativecommons.org/licenses/by/4.0/deed.de) veröffentlicht, welche die Nutzung, Vervielfältigung, Bearbeitung, Verbreitung und Wiedergabe in jeglichem Medium und Format erlaubt, sofern Sie den/die ursprünglichen Autor(en) und die Quelle ordnungsgemäß nennen, einen Link zur Creative Commons Lizenz beifügen und angeben, ob Änderungen vorgenommen wurden.

Die in diesem Kapitel enthaltenen Bilder und sonstiges Drittmaterial unterliegen ebenfalls der genannten Creative Commons Lizenz, sofern sich aus der Abbildungslegende nichts anderes ergibt. Sofern das betreffende Material nicht unter der genannten Creative Commons Lizenz steht und die betreffende Handlung nicht nach gesetzlichen Vorschriften erlaubt ist, ist für die oben aufgeführten Weiterverwendungen des Materials die Einwilligung des jeweiligen Rechteinhabers einzuholen.

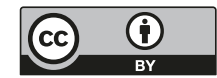

